Bond University

Research Repository

\title{
Do Endoscopic Bariatric Procedures Improve Postprocedural Quality of Life and Mental Health? A Systematic Review and Meta-analysis
}

Gadd, Nicola; McIntosh, Ashleigh; Fear-Keen, Brianna ; Hoult, Jennifer; Maimone, Isabella; Marshall, Skye

Published in:

Obesity Surgery

DOI:

$10.1007 / \mathrm{s} 11695-020-04860-2$

Licence:

Other

Link to output in Bond University research repository.

Recommended citation(APA):

Gadd, N., McIntosh, A., Fear-Keen, B., Hoult, J., Maimone, I., \& Marshall, S. (2020). Do Endoscopic Bariatric Procedures Improve Postprocedural Quality of Life and Mental Health? A Systematic Review and Meta-analysis. Obesity Surgery, 30(10), 4091-4100. https://doi.org/10.1007/s11695-020-04860-2

\section{General rights}

Copyright and moral rights for the publications made accessible in the public portal are retained by the authors and/or other copyright owners and it is a condition of accessing publications that users recognise and abide by the legal requirements associated with these rights.

For more information, or if you believe that this document breaches copyright, please contact the Bond University research repository coordinator. 


\section{Journal: Obesity Surgery}

Do endoscopic bariatric procedures improve postprocedural quality of life and mental health? A systematic review and meta-analysis

Running heading: QoL after bariatric procedures

Nicola Gadd ${ }^{1 *}$, Ashleigh McIntosh ${ }^{2 *}$, Brianna Fear-Keen ${ }^{3}$, Jennifer Hoult ${ }^{4}$, Isabella R Maimone $^{5}$, Skye Marshall ${ }^{6,7}$

1. BHealthSc, MastersNut\&Diet, Faculty of Health Sciences and Medicine, Bond University, Queensland, Australia, nicola.gadd@student.bond.edu.au. ORCID ID: 0000-0002-3014-2929

2. BFood\&NutrSc, MastersNut\&Diet, Faculty of Health Sciences and Medicine, Bond University, Queensland, Australia, ashleigh.mcintosh@student.bond.edu.au. ORCID ID: 0000-0003-2648-0324

3. BNutr\&Diet (Hons), Accredited Practising Dietitian, Weightloss Solutions, Queensland, Australia, Australia, brianna@wlsa.com.au

4. MastersNutr\&Diet, Accredited Practising Dietitian, Weightloss Solutions Australia, Queensland, Australia, dietitian@wlsa.com.au

5. BNutr\&Diet (Hons), Weightloss Solutions Australia, Queensland, Australia, supportdietitian@wlsa.com.au. ORCID ID: 0000-0002-7098-853

6. Bond University Nutrition \& Dietetics Group, Faculty of Health Sciences and Medicine, Bond University, Queensland, Australia, skye_marshall@bond.edu.au; ORCID ID: 0000-0001-8953-5068.

7. Nutrition Research Australia, Sydney, New South Wales, Australia.

*These authors contributed equally to this review.

Corresponding author: Bond University, University Drive, Robina, Queensland, 4226, Australia.skye_marshall@bond.edu.au, +61 (0) 755954410

Key words: Quality of life, intragastric balloon, endoscopic bariatric therapy, mental health, obesity, transpyloric shuttle, primary obesity surgery endoluminal, endoscopic sleeve gastroplasty, aspiration therapy, trans-oral gastroplasty, duodenal bypass liner Word count limits: main text: 4,000, abstract: 200

Word count: main text: 2,983; abstract: 199 
33 Author contributions: NG and AM drafted the manuscript and led the search, data 34 extraction, critical appraisals, meta-analysis, and GRADE assessment. JH, BFK, and IM 35 contributed to data checking and critical appraisals. SM contributed to critical appraisals, 36 meta-analysis, and GRADE assessment. All authors contributed to study concept and 37 manuscript revision.

\section{Conflict of interest}

39 The authors declare there are no conflict of interest.

\section{Acknowledgements}

41 The authors acknowledge the contributions of Bronwyn Linthwaite, Bond University 42 Librarian, for her assistance with the search strategy.

\section{$43 \quad$ Funding}

44 This review has received no specific funding. SM is supported by a Commonwealth of 45 Australia Innovation Connections grant. 


\section{Abstract}

49 Quality of life and mental health are important outcomes of bariatric therapy. This review

50 aimed to determine endoscopic bariatric procedures impact on postprocedural quality of

51 life and mental health. Four electronic databases were systematically searched. Studies

52 with adults $\geq 18$ years who underwent an endoscopic bariatric procedure and reported pre-

53 and postprocedural quality of life and/or mental health using a validated tool were

54 included. Meta-analyses were conducted RevMan and study quality was assessed.

55 Twenty studies evaluating five different endoscopic procedures were included $(\mathrm{N}=876$

56 total sample size). Intragastric balloon placement was associated with a large

57 improvement in postprocedural quality of life and mental health. Endoscopic bariatric

58 therapies may improve short term quality of life and mental health alongside weight loss

59 and comorbidity improvement.

\section{Keywords:}

61 Quality of life, mental health, endoscopic, bariatric.

62

63 


\section{Introduction}

65 Global obesity rates have nearly tripled since 1975 and have been associated with increased incidence of chronic diseases including type 2 diabetes, sleep apnoea, and cardiovascular disease [1]. Obesity and obesity-related stigmatisation also negatively impact on mental health and quality of life (QoL), particularly self-esteem, depression, anxiety, and fear of criticism by others [2, 3]. Weight loss options include traditional lifestyle approaches and bariatric surgeries, such as the gastric bypass, and more recently endoscopic weight loss procedures. These non-surgical procedures have increased from $2 \%$ to $4 \%$ of all bariatric procedures from just 2014 to 2016 [4]. Whilst bariatric surgery has emerged as the most effective long-term method for weight loss, some adults do not prefer this option which is associated with surgical complications (up to $15 \%$ ), morbidity (3-20\%), and mortality (0.1-0.5\%) [5]. Furthermore, some adults with obesity are ineligible for surgery due to operative risks, cardiovascular complications, or a BMI of $\leq 35 \mathrm{~kg} / \mathrm{m}^{2}$ without comorbidities [6-8]. The rise in popularity of endoscopic bariatric procedures reflects their ability to meet such gaps $[9,10]$.

Endoscopic devices currently approved by the United States Food and Drug Administration include gastric balloon (IGB) systems, gastric emptying devices, and other space occupying devices [11]. Other endoscopic bariatric therapies reported in the literature include the transoral gastroplasty, duodenal-jejunal bypass liner, and endoscopic sleeve gastroplasty (ESG) $[9,12,13]$. Mechanisms of action of these endoscopic therapies and devices include gastric restriction, malabsorption, and/or delayed gastric emptying [14].

The weight loss and medical benefits of endoscopic devices have been reported; however,

87 the impacts of endoscopic bariatric procedures on the patient-centred outcomes QoL and mental health are not as well understood [15-19]. The impact of weight loss procedures on QoL is seen by patients as a vital to a successful outcome [3]. The concept of quality 
90 of life encompasses the physical body as well as emotional and social functioning, linking

91 it inherently to mental health [20].

92 As the use of endoscopic bariatric therapies is increasing internationally, there is a need

93 to understand their full impact on candidates by looking beyond weight loss to quality of

94 life and mental health $[16,21]$. Such evidence could enhance the patient-centredness of

95 procedure selection, care planning, and outcome evaluation [22].

$96 \underline{\text { Research question }}$

97 What is the effect of endoscopic bariatric procedures on postprocedural QoL and mental 98 health of adult patients?

\section{Method and Materials}

100 Protocol and registration

101 A systematic review of literature was undertaken and reported according to the PRISMA

102 guidelines [23]. The protocol was prospectively registered with the International

103 Prospective Register for Systematic Reviews (PROSPERO number:

104 CRD42020159822).

\section{$105 \quad$ Eligibility criteria}

106 Studies which included adults $\geq 18$ years who elected an endoscopic bariatric procedure

107 were eligible if they measured pre- and postprocedural QoL or mental health via a

108 validated tool. The following endoscopic bariatric therapies were included in this review:

109 ESG, IGB, transpyloric shuttle (TPS), primary obesity surgery endoluminal (POSE),

110 duodenal-jejunal bypass liner (DJBL), aspiration therapy, duodenal mucosal resurfacing,

111 incisionless anastomosis, overstitching endoscopic suturing system, transoral

112 gastroplasty, and transoral endoscopic restrictive implant system. Studies were excluded

113 when endoscopic therapy data was merged with excluded therapies including medical,

114 lifestyle, and/or surgical weight loss. This review considered original research studies 
115 including prospective and retrospective observational studies and intervention studies;

116 studies were not limited by publication date. Each eligible arm of an intervention study

117 was considered alone (i.e. not in relation to the comparator group) as representing

118 prospective cohort data. Intervention study arms (whether comparator or intervention)

119 which provided additional counselling and/or postprocedural variations in support

120 beyond usual care, which would affect QoL and mental health outcomes, were excluded.

121 Studies were sourced in any language if they could be translated to English using Google

122 Translate [24]. Excluded publication types were conference abstracts or papers, reviews,

123 study protocols, cross-sectional studies, and qualitative studies.

\section{$124 \quad$ Search strategy and study selection}

125 Studies were searched in the electronic databases: EMBASE, Medline, CINAHL, and

126 PsycINFO. The search strategy comprised a combination of controlled vocabulary and

127 keywords (Table S1). The search strategy was designed in PubMed and translated into

128 other databases using the Systematic Review Accelerator Polyglot Search [25]. A

129 structured sensitivity analysis of the search strategy was undertaken in EMBASE and

130 CINAHL. When studies were irretrievable corresponding authors were contacted. Alerts

131 for new studies were set up across databases, with any new eligible studies included up

132 until $21^{\text {st }}$ March 2020. Reference lists of relevant papers were hand searched to identify

133 additional studies. Systematic search results were de-duplicated with Systematic Review

134 Accelerator De-Duplicate software [25, 26]. Covidence software was utilized for 135 screening of title/abstract and full text and was undertaken independently by two 136 reviewers (AM and $\mathrm{NG)}$ [27]. A third reviewer (SM) assisted with eligibility

137 disagreements. Corresponding authors were contacted for studies requiring further 138 information to determine eligibility. 
140 Outcomes were QoL, depression, anxiety, and mood. Outcomes which were considered

141 as confounding variables on the primary outcomes included: changes in body

142 composition (excess weight loss [EWL], body mass index [BMI], total body weight, fat

143 mass, or waist circumference), changes in incidence or prevalence of comorbidities, and

144 peri- and postprocedural adverse events.

\section{Data extraction}

146 Data were extracted a single investigator (AM or NG) and checked for accuracy by a

147 second (JH, BFK, or IM). Any corrections to extracted data by the second reviewer were

148 verified by a third investigator (NG or AM). For studies with missing data, corresponding

149 authors were contacted. Where data on the same study variable was reported in multiple

150 publications, the data extracted comprised either the most complete data (e.g. that which

151 reported variance), data representing intention to treat analysis, or the largest sample size.

152 Data reported in graphical form was extracted via Web Plot Digitizer software [28].

\section{Quality assessment and risk of bias}

154 Included articles were critically appraised by two investigators independently (AM and

155 NG) using the Academy of Nutrition and Dietetics Quality Criteria Checklist [29].

156 Studies were rated as positive, neutral, or negative quality based on the internal risk of

157 bias. Disagreements were resolved through discussion until consensus was reached and

158 decision making was reviewed by a third authors (SM).

159 GRADEpro software was used to rate the confidence in the body of evidence for all

160 studies with a primary outcome. Confidence in the body of evidence considered study

161 design, risk of bias, consistency, directness, publication bias, effect sizes, and precision

162 according to the Grading of Recommendations Assessment, Development and Evaluation 
163 methodology (GRADE) approach (Table S4) [30]. GRADE was completed initially by

164 AM and through consensus of three authors (AM, NG, SM).

\section{$165 \quad$ Meta-analytical approach}

166 Ccontinuous data were pooled using the inverse variance test using RevMan (Review

167 Manager 5, Version 5.3) [31]. The total QoL score was prioritized and when not available, 168 general health domain was used. When standard deviations were not reported, a 169 calculation was made using the RevMan Calculator (Review Manager 5, Version 5.3). If 170 data were presented as median (interquartile range), the distance between the interquartile 171 range was assumed to be 1.35 standard deviations [32]. Outcomes were reported as 172 standardized mean differences (SMD) to account for the different tools used to measure 173 each construct. A random effects model was used across all meta-analytical models 174 representing the substantial clinical heterogeneity expected. Studies were assessed for 175 statistical consistency using the $\mathrm{I}^{2}$ statistic. High levels of statistical inconsistency were 176 explored using confounding variables, outlier results, or sample characteristics in a 177 sensitivity analysis.

\section{Results}

179 Search results and study characteristics

180 The search strategy retrieved 5,959 records, 338 records were full text screened for

181 eligibility, and 20 papers were included (Figure 1). Two additional records were identified

182 through snowballing. The main reason for exclusion was study design $(\mathrm{n}=146)$ and 183 surgical bariatric therapy $(\mathrm{n}=134)$.

184 The 20 studies were published between 2008 and 2019 with a total number of 876 patients 185 (77\% female). Intragastric balloons were the predominant endoscopic therapy $(n=14)[2$, $18620,33-45]$, followed by aspiration therapy $(n=2)[46,47]$, TOGA $(n=2)[12,48]$, ESG $187(\mathrm{n}=1)$ [13], and TPS $(\mathrm{n}=1)$ [49] (Table S2). 
188 Most studies were observational studies $(n=15)$, with the remainder being randomised

189 controlled trials (RCTs) $(n=5)$. All studies were rated as positive quality $(n=9)$ or neutral

190 quality ( $\mathrm{n}=11)$ (Table S3). The most common reasons for downgrading the quality of

191 studies were failing to report eligibility criteria or sampling method, insufficient duration

192 of intervention, or failure to account for confounding factors in the statistical analysis.

193 The overall GRADE for QoL and mental health was "low" and "very low" due to the

194 majority of the studies using a prospective observational design as opposed to randomised

195 controlled trials, some risk of bias, and statistical inconsistency (Table S4).

196 Endoscopic bariatric therapies' impact on quality of life

197 All but one study measured QoL ( $\mathrm{n}=19$ studies) using a range of tools (Table 1). Eighteen

198 studies reported a statistically significant improvement in QoL from baseline to follow-

199 up, with only one study showing no change [44]. Interestingly, three studies appear to

200 have misinterpreted their QoL results [36, 39, 42].

201 Nine studies with a total of 371 participants ( $n=350$ at follow-up) who underwent IGB

202 (6- to 76-month follow-up) were included via meta-analysis. Intragastric balloon

203 placement was associated with a significant improvement in QoL (SMD:0.78; 95\%CI:

$\left.2040.56,1.00 ; \mathrm{P}=0.05 ; \mathrm{I}^{2}: 48 \%\right)$. A sensitivity analysis identified that results from De Castro

205 et al 2010 [44] impacted on the overall $\mathrm{I}^{2}$ and was removed in sensitivity analysis on the

206 basis of QoL construct differences. Specifically, De Castro et al 2010 [44] used the GIQLI

207 tool which assesses gastrointestinal-related QoL whereas other studies assessed general

208 health-related QoL. Following sensitivity analysis, IGB placement was associated with a

209 large improvement in postprocedural QoL (SMD: 0.85; 95\%CI: 0.69, 1.02; P $<0.00001$;

$210 \quad I^{2}: 7 \%$; Figure 2). Insufficient data prevented other endoscopic bariatric therapies' impact

211 on QoL being pooled via meta-analysis. It was not possible to assess publication bias due

212 to small number of studies included in the meta-analysis. 


\section{Endoscopic bariatric therapies' impact on mental health}

214 Depression, anxiety, and/or mental health including psychological or emotional health

215 were assessed in seven studies, six of which excluded patients with psychiatric disorders

216 or those taking anti-depressants [2, 41, 42, 45-47]. (Table S2). All studies reported a

217 statistically significant postprocedural improvement in mental health. Five IGB studies

218 were pooled via meta-analysis ( $n=367$ participants at 6 to 76 -months follow-up), finding

219 that IGB was associated with a large improvement in the mental health, depression, or

220 anxiety (SMD: $0.86 ; 95 \% \mathrm{CI}: 0.29,1.42 ; \mathrm{P}=0.003 ; \mathrm{I}^{2}=92 \%$; Figure 3 ). Insufficient data

221 prevented other endoscopic bariatric therapies' impact on mental health being pooled via

222 meta-analysis. It was not possible to assess publication bias due to small number of

223 studies included in the meta-analysis.

224 Impact of confounding factors on quality of life and mental health

225 All studies in the meta-analysis were neutral quality except two studies [2, 20]. Studies reporting the most significant changes in QoL and mental health were rated neutral [34, 40]. All studies reported a significant decrease in weight as changes to total body weight, BMI, TBWL\%, or EWL\%. The two studies (Guedes et al 2019 and Deliopoulo et al 2013) with the largest improvements in mental health also had the greatest weight loss $[40,43]$; however, associations with strength of weight loss and change in mental health were not consistent thereafter. The largest improvements in QoL did not coincide with the highest mean weight loss. Guedes et al 2019 [40] reported the largest weight loss but only a small improvement in QoL. However, Tayyem et al 2014 [34] and Fuller et al 2013 [42] had

234 slightly less but very similar weight loss to Guedes et al 2019 [40] and reported the most significant improvements in QoL.

236 Improvements in one or more comorbidities at follow-up were reported in nine studies

237 including significant improvements and/or remission of type II diabetes mellitus, 
238 hypertension, obstructive sleep apnoea, and metabolic syndrome [12, 13, 20, 33, 39-41,

239 46-48]. Studies that reported comorbidity risk factors (blood pressure, HbA1c,

240 triglycerides, or LDL cholesterol) also reported improvements (Table S2). Three studies

241 did not report follow-up comorbidity data $(34,40,48)$. No association was seen between

242 improvements in comorbidities and improvements in QoL or mental health.

243 Adverse events were reported categorically as ordinal or nominal variables or as a reason

244 for study withdrawal in 13 studies [2, 20, 33, 36-39, 41, 42, 44, 46-49]. The most common

245 adverse events were nausea and vomiting. Early balloon removal occurred in three

246 studies: 1.2\% in Alfredo et al 2014 [41], 3.4\% in Mui et al 2010 [20] and 22\% in Guedes

247 et al 2017 [2]. Although the impact of adverse events on QoL in De Castro et al 2010 [44]

248 was evident, there was no other clear associations found between adverse events and

249 mental health or QoL.

250 The amount and type of multidisciplinary support provided to patients varied and was

251 only reported in 10 of the 20 studies [2, 20, 37-39, 41-43, 46, 47]. Types of support

252 included: unlimited 24 hour phone support [43], follow-up with a dietitian [2, 20, 41, 43],

253 nutrition counselling [38, 39, 47], cognitive behavioural therapy [47], and/or a lifestyle

254 modification program [42, 46]. Studies with the most significant improvements in mental

255 health and QoL provided patients with the most support [2, 20, 42, 43]

256 Discussion

257 This systematic review and meta-analysis evaluated the effects of endoscopic bariatric 258 procedures on postprocedural QoL and mental health using mostly observational

259 evidence. Qualitative synthesis found strong and consistent improvements in QoL (95\%

260 of studies) and mental health (100\% of studies). Meta-analyses of IGB studies also

261 showed large statistically significant improvements in QoL and mental health. Pooled

262 findings showed strong consistency for QoL; however, there was statistical inconsistency 
in pooled effects on mental health, likely due to slight differences in the concepts included

264 in mental health assessment tools. Although pooled effect sizes were large for the impact of IGB on postprocedural QoL and mental health; confidence in the body of evidence was low and very low respectively, where main reasons for downgrading were related to risk

267 of bias in the included studies and the observational study design, highlighting the need 268 for further RCTs.

269 A systematic review by Lindekilde et al [50] evaluated the impact of any bariatric 270 procedure (mostly surgical, two were endoscopic), reporting similar improvements in 271 postprocedural QoL. The previous review found an association of positive changes in 272 QoL with higher weight loss [50]. However, the current review did not find a consistent positive association between weight loss and quality of life. The drivers of improvements in QoL following endoscopic bariatric procedures may be necessarily be due to the amount of weight loss alone and is likely to also reflect changes in physical appearance and physical function, general health through improvements in comorbidities, and social functioning due to increased confidence $[12,35]$. Although this study did not identify an association between quality of life or mental health with improved comorbidities, this is likely a reflection of comorbid outcomes being inconsistently measured. Gastrointestinalrelated QoL seems to differ from other postprocedural QoL domains. This review found much smaller and/or no improvements in gastrointestinal-related QoL, likely related to commonly reported gastrointestinal adverse events by studies using endoscopic bariatric procedures [50].

The reported improvements in mental health found in this review also align with the findings of Dawes et al [51], which evaluated the impact of bariatric surgery on mental health. Spirou et al [16] also found similar results at six-months postoperative; although, results at $\geq 36$-months showed a reduction in mental health improvements. These findings suggested that QoL and mental health improvements may not be retained long-term and 
may be due to a 'psychological honeymoon period' due to initial weight loss [16]. This

290 may be translatable to endoscopic bariatric procedures, many of which are temporary. An

291 association with weight change and mental health was identified in this review, which is

292 inconsistent with previous research. Results suggest the amount of weight lost positively

293 impacted participants mental health change; each study displayed a significant decrease

294 in weight following endoscopic procedures. Canetti et al [52] analysed the change in

295 mental health and QoL in Silastic Ring Vertical Banded Gastroplasty (laparoscopic)

296 patients. Findings showed even though weight loss at 10 -years was maintained,

297 improvements in mental health were not.

298 The study with the most significant improvement in mental health and weight loss offered

299 24-hour telephone support and monthly dietitian follow-ups. A recent systematic review

300 and meta-analysis found that compared with standard multidisciplinary care, intensive

301 pre- and/or postoperative psychological intervention resulted in significantly improved

302 postoperative symptoms of depression and anxiety [53]. This suggests that while bariatric

303 procedures, whether endoscopic or surgical, may improve mental health at least

304 temporarily, the greatest improvements are seen with intensive multidisciplinary support,

305 aligning with bariatric clinical practice guidelines [54].

$306 \quad \underline{\text { Limitations }}$

307 Meta-analysis was limited by the number of diverse endoscopic procedures which have

308 measured and adequately reported postprocedural QoL and mental health. Conclusions

309 are also limited by the short duration of follow-up; meaning results cannot be interpreted

310 to represent long-term outcomes. The meta-analysis was unable to control for variations

311 of the effect of the procedure and confounding characteristics [55]. The exclusion of

312 patients with psychiatric disorders or those taking anti-depressants limits the

313 generalisability of the findings on mental health. Confidence that the estimated pooled

314 means reflect the true change in QoL and mental health is low and very low. Therefore, 
315 findings must be interpreted with the understanding that they may change with the

316 availability of higher quality evidence, such as that from well conducted RCTs with

317 adequate blinding and length of follow-up.

\section{Implications for future practice and research}

319 Health professionals should recognise the importance of QoL and mental health to 320 patients and provide multidisciplinary support in line with the latest clinical practice 321 guidelines [55], which includes dietetic and psychological intervention. Future research

322 should be improved by strengthening the reporting of methods and results by utilising 323 validated checklists such as the STROBE checklist for observational studies [56]. Studies

324 should also seek to always contain patient-centred outcomes such as QoL, mental health, 325 and the effects of weight stigmatisation in addition to clinical weight loss and medical 326 outcomes. Consideration should be given to the reporting of results, including the 327 reporting of baseline, change, and follow up measures of central tendency and variance, 328 and not report results only graphically. Future research should incorporate QoL and 329 mental health as an integral outcome of therapy success with further examination of 330 weight-stigma.

\section{Conclusion}

332 Endoscopic bariatric procedures, particularly IGB, may improve postprocedural QoL and 333 mental health alongside weight loss and comorbidity improvements; however, their effect 334 on long term QoL and mental health is unknown. Multidisciplinary support by dietitians 335 and/or psychologists is important for optimising QoL and mental health outcomes.

336 Further research is required to understand the impact of diverse types endoscopic bariatric 337 procedures on QoL and mental health in the long term.

\section{Ethical approval}


339 This article does not contain any studies with human participants or animals performed

340 by any of the authors.

\section{Conflict of interest}

342 The authors declare there are no conflict of interest.

343

344 Abbreviations

345 BAROS, Bariatric Analysis and Reporting Outcome System

346 BQL, Body Quality of Life

347 DJBL, Duodenal-jejunal bypass liner

348 ESG, Endoscopic Sleeve Gastroplasty

349 EWL, Excess Weight Loss

350 FDA, Food and Drug Administration

351 GIQLI, Gastrointestinal Quality of Life

352 IGB, Intragastric Balloon

353 OR, Odds Ratio

354 POSE, Primary obesity surgery and endoluminal

355 QoL, Quality of life

356 SF-36, Short Form Health Survey

357 SMD, Standardised Mean Difference

358 TPS, Transpyloric Shuttle 
360 Conflict of interest

361 The authors declare there are no conflict of interest.

362 Ethical approval statement

363 This article does not contain any studies with human participants or animals performed

364 by any of the authors.

365 Informed consent.

366 Informed consent does not apply. 


\section{References}

1. World Health Organization. Obesity and overweight. . Available from:

https://www.who.int/news-room/fact-sheets/detail/obesity-and-overweight. Published 2018. Acessed November 11, 2019.

2. Guedes, E., et al., Impact of 6 months of treatment with intragastric balloon on body fat and quality of life in obese individuals with metabolic syndrome. Health and Quality of Life Outcomes, 2017. 15(1): p. 1-6.

3. Sierżantowicz, R., et al., Effect of BMI on quality of life and depression levels after bariatric surgery. Advances in clinical and experimental medicine : official organ Wroclaw Medical University, 2017. 26(3): p. 491.

4. Angrisani, L., et al., IFSO Worldwide Survey 2016: Primary, Endoluminal, and Revisional Procedures. Obesity Surgery, 2018. 28(12): p. 3783-3794.

5. Buchwald, H., et al., Bariatric surgery: A systematic review and meta-analysis. JamaJournal Of The American Medical Association, 2004. 292(14): p. 1724-1737.

6. Regan, J., et al., Early Experience with Two-Stage Laparoscopic Roux-en-Y Gastric Bypass as an Alternative in the Super-Super Obese Patient. Obesity Surgery, 2003. 13(6): p. 861-864.

7. Schwartz, A., L. Etchechoury, and D. Collet, Outcome after laparoscopic gastric bypass for super-super obese patients. Journal of Visceral Surgery, 2013. 150(2): p. 145-149.

8. Villamere, J., et al., Body mass index is predictive of higher in-hospital mortality in patients undergoing laparoscopic gastric bypass but not laparoscopic sleeve gastrectomy or gastric banding. American Surgeon, 2014. 80(10): p. 1039-1043.

9. Behary, J. and V. Kumbhari, Advances in the Endoscopic Management of Obesity. Gastroenterology Research and Practice, 2015. (2015): 1-9. 
10. Abu Dayyeh, B.K., et al., ASGE Bariatric Endoscopy Task Force systematic review and meta-analysis assessing the ASGE PIVI thresholds for adopting endoscopic bariatric therapies. Gastrointest Endoscopy, 2015. 82(3): p. 425-38.e5.

11. FDA (Food and Drug Adminitsration). Weight-Loss and Weight Management Devices. 2019 [cited 2020 11-03]; Available from: https://www.fda.gov/medicaldevices/products-and-medical-procedures/weight-loss-and-weight-managementdevices\#loss.

12. Familiari, P., et al., Transoral gastroplasty for morbid obesity: a multicenter trial with a 1-year outcome. Gastrointestinal Endoscopy, 2011. 74(6): p. 1248-1258.

13. Fiorillo, C., et al., 6-Month Gastrointestinal Quality of Life (QoL) Results after Endoscopic Sleeve Gastroplasty and Laparoscopic Sleeve Gastrectomy: A Propensity Score Analysis. Obes Surg, 2020.

14. Jason, B. and K. Vivek, Advances in the Endoscopic Management of Obesity.

Gastroenterology Research and Practice, 2015. 2015(2015).

15. Saber, A.A., et al., Efficacy of First-Time Intragastric Balloon in Weight Loss: a Systematic Review and Meta-analysis of Randomized Controlled Trials. Obes Surg, 2017. 27(2): p. 277-287.

16. Spirou, D., J. Raman, and E. Smith, Psychological outcomes following surgical and endoscopic bariatric procedures: A systematic review. Obes Rev, 2020.

17. Yorke, E., et al., Intragastric Balloon for Management of Severe Obesity: a Systematic Review. Obes Surg, 2016. 26(9): p. 2248-2254.

18. Fernandes, M.A.P., et al., Intragastric balloon for obesity. Cochrane Database of Systematic Reviews, 2007(1). 
19. Szmulewicz, A., et al., Mental health quality of life after bariatric surgery: A systematic review and meta-analysis of randomized clinical trials. Clinical obesity, 2019. 9(1): p. e12290.

20. Mui, W., et al., Impact on Obesity-Related Illnesses and Quality of Life Following Intragastric Balloon. Obesity Surgery, 2010. 20(8): p. 1128-1132.

21. NCE (National Institute for Health and Care Excellence) Obesity: identification, assessment and management. 2014.

22. Accardi, R., et al., Italian version of the laval questionnaire: Validity and reliability. Bariatric Surgical Practice and Patient Care, 2017. 12(3): p. 136-141.

23. Moher, D., et al., Preferred reporting items for systematic reviews and meta-analyses: the PRISMA statement. Annals of internal medicine, 2009. 151(4): p. 264.

24. Google. Google Translate. Available from: https://translate.google.com/. Acessed November 11, 2019.

25. Bond University. Systematic Review Accelerator.; Available from: http://sraccelerator.com/\#/. Acessed November 11, 2019.

26. EndNote [computer program]. Version X8, Web of Science Group. 2019.

27. Covidence systematic review software, Veritas Health Innovation, Melbourne, Australia. Available at www.covidence.org.

28. PLOTCON, WebplotDigitizer. 2017: Oakland, CA.

29. Academy of Nutrition \& Dietetics. Evidence Analysis Manual. Appendix 8: Quality Criteria Checklist: Primary Research Available from: https://www.andeal.org/evidence-analysis-manual Published 2016. Accessed October 28,2019 
30. Erika Paniago, G., et al., Impact of 6 months of treatment with intragastric balloon on body fat and quality of life in obese individuals with metabolic syndrome. Health and Quality of Life Outcomes, 2017. 15(1): p. 1-6.

31. The Cochrane Collaboration, Review Manager [computer program]. Version 5.3. 2014: Copenhagen: The Nordic Cochrane Centre; .

32. Higgins, J.P.T., Cochrane Handbook for Systematic Reviews of Interventions. 2nd ed. ed. Wiley Cochrane Ser., ed. J. Thomas. 2019, Newark: John Wiley \& Sons, Incorporated.

33. Tayyem, R.M., C. Obondo, and A. Ali, Short-term outcome and quality of life of endoscopically placed gastric balloon and laparoscopic adjustable gastric band. Saudi journal of gastroenterology : official journal of the Saudi Gastroenterology Association, 2011. 17(6): p. 400.

34. Tayyem, R.M., J.M. Atkinson, and C.R. Martin, Development and validation of a new bariatric-specific health-related quality of life instrument "bariatric and obesityspecific survey (BOSS)". Journal of postgraduate medicine, 2014. 60(4): p. 357.

35. Guedes, E., et al., Impact of a 6-month treatment with intragastric balloon on body composition and psychopathological profile in obese individuals with metabolic syndrome. Diabetology and Metabolic Syndrome, 2016. 8(1): p. 1-7.

36. Raftopoulos, I. and A. Giannakou, The Elipse Balloon, a swallowable gastric balloon for weight loss not requiring sedation, anesthesia or endoscopy: a pilot study with 12month outcomes. Surgery for Obesity and Related Diseases, 2017. 13(7): p. 11741182.

37. Reimão, S., et al., Improvement of Body Composition and Quality of Life Following Intragastric Balloon. Obesity Surgery, 2018. 28(6): p. 1806-1808. 
38. Ponce, J., B.B. Quebbemann, and E.J. Patterson, Prospective, randomized, multicenter study evaluating safety and efficacy of intragastric dual-balloon in obesity. Surgery for Obesity and Related Diseases, 2013. 9(2): p. 290-295.

39. Machytka, E., et al., Elipse, the first procedureless gastric balloon for weight loss: a prospective, observational, open-label, multicenter study. Endoscopy, 2017. 49(2): p. 154.

40. Guedes, M.R., et al., Changes in Body Adiposity, Dietary Intake, Physical Activity and Quality of Life of Obese Individuals Submitted to Intragastric Balloon Therapy for 6 Months. Obesity surgery, 2019. 29(3): p. 843.

41. Alfredo, G., et al., Long-term multiple intragastric balloon treatment-a new strategy to treat morbid obese patients refusing surgery: Prospective 6-year follow-up study. Surgery for Obesity and Related Diseases, 2014. 10(2): p. 307-311.

42. Fuller, N., et al., An intragastric balloon in the treatment of obese individuals with metabolic syndrome: A randomized controlled study. Obesity, 2013. 21(8): p. 15611570.

43. Deliopoulou, K., et al., The Impact of Weight Loss on Depression Status in Obese Individuals Subjected to Intragastric Balloon Treatment. Obesity Surgery, 2013. 23(5): p. 669-675.

44. Castro, M., et al., Efficacy, Safety, and Tolerance of Two Types of Intragastric Balloons Placed in Obese Subjects: A Double-Blind Comparative Study. Obesity Surgery, 2010. 20(12): p. 1642-1646.

45. Ahmed, H.O. and R.F. Ezzat, Quality of life of obese patients after treatment with the insertion of intra-gastric balloon versus Atkins diet in Sulaimani Governorate, Kurdistan Region, Iraq. Annals of medicine and surgery (2012), 2018. 37: p. 42-46. 
46. Christopher, C.T., et al., Percutaneous Gastrostomy Device for the Treatment of Class II and Class III Obesity: Results of a Randomized Controlled Trial. The American Journal of Gastroenterology, 2016. 112(3).

47. Norén, E. and H. Forssell, Aspiration therapy for obesity; a safe and effective treatment. BMC obesity, 2016. 3(1).

48. Moreno, C., et al., Transoral gastroplasty is safe, feasible, and induces significant weight loss in morbidly obese patients: results of the second human pilot study. Endoscopy, 2008. 40(5): p. 406.

49. Marinos, G., et al., Weight loss and improved quality of life with a nonsurgical endoscopic treatment for obesity: clinical results from a 3-and 6-month study. Surgery for Obesity and Related Diseases, 2014. 10(5): p. 929-934.

50. Lindekilde, N., et al., The impact of bariatric surgery on quality of life: a systematic review and meta-analysis. 2015. p. 639-651.

51. Dawes, A.J., et al., Mental Health Conditions Among Patients Seeking and Undergoing Bariatric Surgery: A Meta-analysis. JAMA, 2016. 315(2): p. 150.

52. Canetti, L., E. Bachar, and O. Bonne, Deterioration of mental health in bariatric surgery after 10 years despite successful weight loss. European journal of clinical nutrition, 2016. 70(1): p. 17.

53. Marshall, S., et al., Does intensive multidisciplinary intervention for adults who elect bariatric surgery improve postoperative weight loss, comorbidities, and quality of life? A systematic review and meta-analysis Obesity Reviews, 2020. In Press.

54. Mechanick, J.I., et al., Clinical practice guidelines for the perioperative nutrition, metabolic, and nonsurgical support of patients undergoing bariatric procedures2019 update: cosponsored by American Association of Clinical Endocrinologists/American College of Endocrinology, The Obesity Society, American 
Society for Metabolic \& Bariatric Surgery, Obesity Medicine Association, and American Society of Anesthesiologists. Surgery for Obesity and Related Diseases, 2019.

55. Cuijpers, P., et al., Pre-post effect sizes should be avoided in meta-analyses. Epidemiology and psychiatric sciences, 2017. 26(4): p. 364.

56. Von Elm, E., et al., The Strengthening the Reporting of Observational Studies in Epidemiology (STROBE) statement: guidelines for reporting observational studies. Preventive Medicine, 2007. 45(4): p. 247-251.

57. Allergan. Intragastric Balloon System—Directions For Use (DFU). 2011; Available from: http://www.allergan.com.au/Products/Overview.aspx.

58. Beck, A.T., et al., An inventory for measuring depression. Archives of general psychiatry, 1961. 4: p. 561.

59. Beck, A.T., R.A. Steer, and G.K. Brown, Beck Depression Inventory - Second Edition (BDI-II). 1996.

60. EuroQol Research Foundation. EQ-5D-5L User Guide. 2019 [cited 2020 10.02]; Available from: https://euroqol.org/publications/user-guides.

61. Eypasch, E., et al., Gastrointestinal Quality of Life Index: development, validation and application of a new instrument. Br J Surg, 1995. 82(2): p. 216-22.

62. Zigmond, A.S. and R.P. Snaith, The Hospital Anxiety and Depression Scale. Acta Psychiatrica Scandinavica, 1983. 67(6): p. 361-370.

63. Organization, W.H. WHO Quality of Life-BREF (WHOQOL-BREF). n.d; Available from: https://www.who.int/substance_abuse/research_tools/whoqolbref/en/.

64. Kolotkin, R. IWQOL-Lite Asessing the impact of weight on quality of life in adults. 2017; Available from: https://www.qualityoflifeconsulting.com/iwqol-lite.html. 
65. Kolotkin, R.L., S. Head, and A. Brookhart, Construct validity of the impact of weight on quality of life questionnaire. Obesity research, 1997(5): p. 434-441.

66. Ware, J.E., M. Kosinski, and S.D. Keller, A 12-Item Short-Form Health Survey: Construction of Scales and Preliminary Tests of Reliability and Validity. Medical Care, 1996. 34(3): p. 220-233.

67. J., W., et al. SF-36 Health Survey Manual and Interpretation Guide. 1993. 

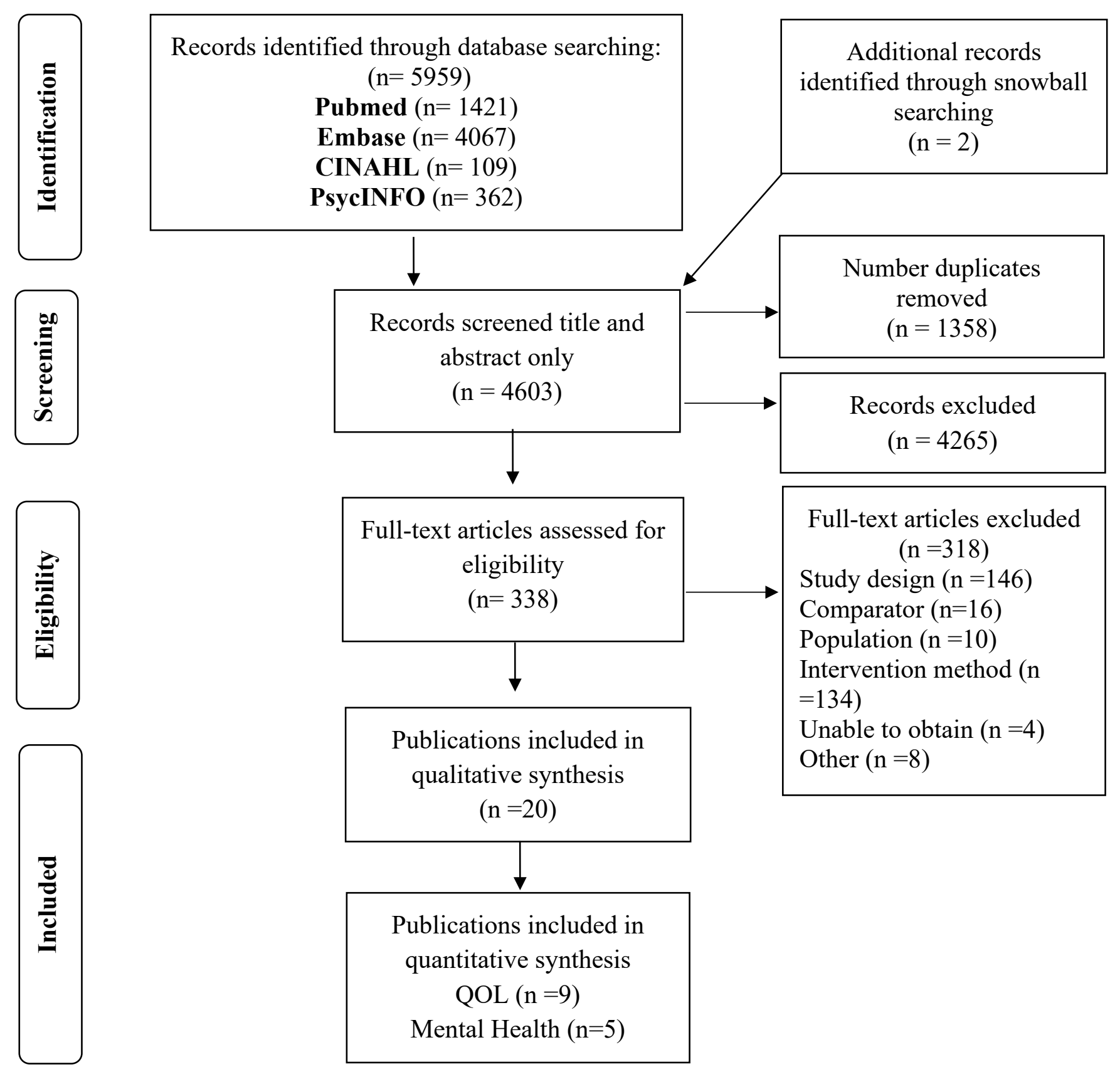

Figure 1: PRISMA diagram for the study 


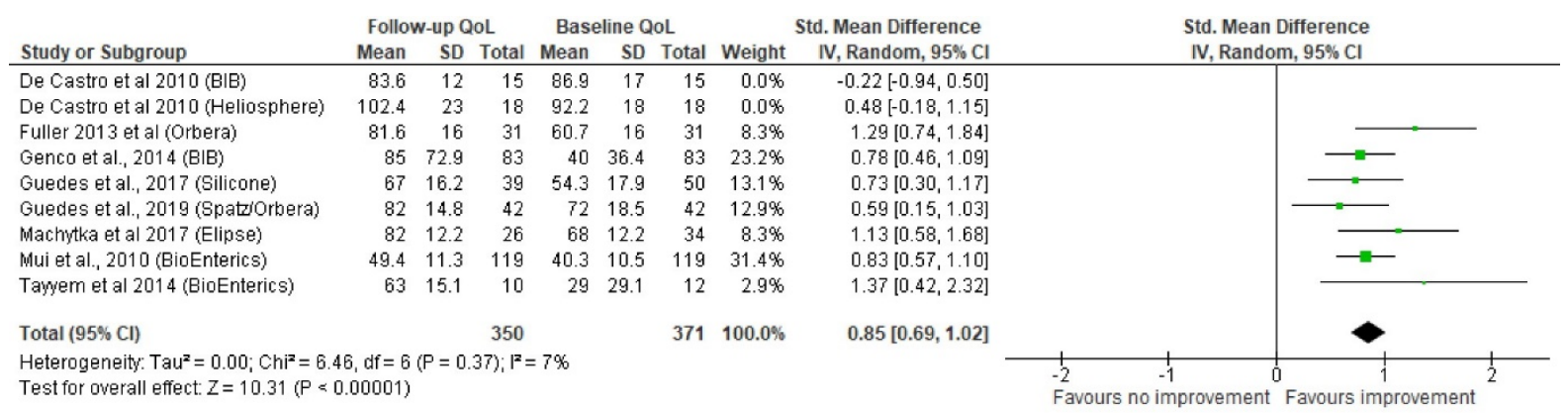

Figure 2. Pooled effects of intragastric balloon placement on pre- to postprocedural quality of life. 


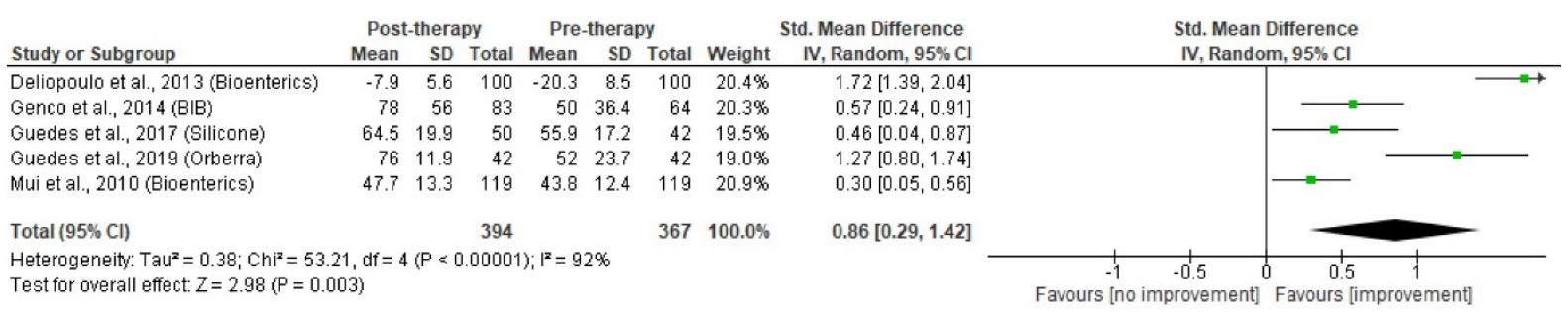

Figure 3. Pooled effects of intragastric balloon placement on pre- to postprocedural mental health 
Table 1. Characteristics and outcomes of the 20 included publications which reported quality of life and/or mental health pre and post endoscopy bariatric therapies in adults.

\begin{tabular}{|c|c|c|}
\hline Study design, setting and participants & Endoscopic therapy & Outcomes \\
\hline $\begin{array}{l}\text { Ahmed et al, } 2019^{[45]} \text {; Prospective study-; } 2008-12 \text {; } \\
\text { Iraq; BIB: } \mathrm{n}=40,100 \% \mathrm{~F}, \mu 27 \mathrm{y} \text { (range: } 20-39 \mathrm{y} \text { ), } \\
\text { BMI } \mu 36 \text { (range: } 31-39.9 \text { ) } \mathrm{kg} / \mathrm{m}^{2}, 0 \% \text { attrition. }\end{array}$ & $\begin{array}{l}\text { BIB; Duration of Tx: } 6 \mathrm{~m} \text {; Method: inserted under } \\
\text { sedation, } 600 \mathrm{~mL} \text { saline containing } 10 \% \text { methyl blue; } \\
\text { Follow-up: } \mathrm{d} 7, \mathrm{~d} 14 \text {, then monthly. }\end{array}$ & Quality of life (6m follow-up): EQ-5D: NR. \\
\hline \multirow{2}{*}{$\begin{array}{l}\text { De Castro et al } 2010^{[44]} ; \text { Prospective study; } 2006-9 \text {; } \\
\text { Spain; BIB: } \mathrm{n}=15,67 \% \mathrm{~F}, \mu 45.4 \pm 8 \mathrm{y}, \mathrm{BMI} \mu 44.2 \pm 6 \\
\mathrm{~kg} / \mathrm{m} 2 \text {. Heliosphere IGB: } \mathrm{n}=18,72 \% \mathrm{~F}, \mu 42.7 \pm 12 \mathrm{y} \\
\text { BMI } \mu 44.2 \pm 5 \mathrm{~kg} / \mathrm{m}^{2}, 18 \% \text { attrition. }\end{array}$} & $\begin{array}{l}\text { BIB; Duration of Tx: } 6 \mathrm{~m} \text {; Method: inserted under } \\
\text { conscious sedation, } 700 \mathrm{ml} \text { saline containing methylene } \\
\text { blue; Follow-up: monthly. }\end{array}$ & $\begin{array}{l}\text { Quality of life (6m follow-up): GIQLI score: baseline } \mu 86.9 \pm 17 \text {. } \\
\text { Follow-up: } \mu 83.6 \pm 12 . \text { Calculated change: }-3.3 \text {. }\end{array}$ \\
\hline & $\begin{array}{l}\text { Heliosphere IGB; Duration of Tx: 6m; Method: } 960 \mathrm{~cm}^{3} \\
\text { air; Follow-up: monthly. }\end{array}$ & $\begin{array}{l}\text { Quality of life (6m follow-up): GIGLI score: baseline } \mu 92.2+18 \text {. } \\
\text { Follow-up: } \mu 102.4 \pm 23 \text {. Calculated change: }+10.2 \text {. }\end{array}$ \\
\hline $\begin{array}{l}\text { Deliopoulo et al, } 2013{ }^{[43]} \text {; Prospective study-; } \\
\text { 2009-2010; Greece; IGB: } \mathrm{n}=100 \text {; Depressed group: } \\
\mathrm{n}=65,100 \% \mathrm{~F}, \mu 37.52+11.77 \mathrm{y} \text { [median: } 37 \text {, range: } \\
\text { 19-61], } \mu 43.5+9.5 \mathrm{~kg} / \mathrm{m} 2 . \text { Non-depressed group: } \\
\mathrm{n}=35,100 \% \mathrm{~F}, 33.89+11.50 \mathrm{y}[33,18-63] \text {, BMI } 41.9 \\
+7.4 \mathrm{~kg} / \mathrm{m}^{2}, 0 \% \text { attrition. }\end{array}$ & $\begin{array}{l}\text { BioEnterics IGB; Duration of Tx: } 6 \mathrm{~m} \text {; Method: inserted } \\
\text { under sedation; Follow-up: monthly }+24 \mathrm{~h} \text { telephone } \\
\text { helpline. }\end{array}$ & $\begin{array}{l}\text { Depression symptoms (6m follow-up): BDI-II baseline: } \mu 20.3+ \\
8.5 \text { [range: } 10-54 \text { ]. Follow-up: } \mu 7.9+5.6 \text { [range: } 0-26], \mathrm{p}<0.0001 \\
\text { Calculated change }=-12.4 .\end{array}$ \\
\hline $\begin{array}{l}\text { Familiari et al, 2011. }{ }^{[12]} \text {; Prospective study-; } 2007- \\
\text { 2010; Italy \& Belgium; TOGA: } \mathrm{n}=67 ; \text { follow-up } \\
\mathrm{n}=53 ; 70 \% \mathrm{~F}, \mu 41.0+9.7 \mathrm{y}, \text { BMI } \mu 41.5+3.6 \mathrm{~kg} / \mathrm{m}^{2} \\
21 \% \text { attrition. }\end{array}$ & $\begin{array}{l}\text { TOGA sleeve stapler \& restrictor systems; Method: } \\
\text { inserted under sedation, sleeve stapler device; Follow- } \\
\text { up: monthly. }\end{array}$ & $\begin{array}{l}\text { Quality of Life (12m follow-up): SF-36v2 \& IWQOL-Lite: } p=< \\
0.001 .\end{array}$ \\
\hline $\begin{array}{l}\text { Fiorillo et al, } 2020^{[13]} \text {; Retrospective study; } 2016- \\
2018 ; \text { France; ESG: } \mathrm{n}=84 ; ; 70 \% \mathrm{~F}, \mu 41 \mathrm{y}(\text { range: } 35- \\
\text { 43y), BMI } \mu 39.5 \text { (range: } 36.7-44.7) \mathrm{kg} / \mathrm{m}^{2}, 50 \% \\
\text { attrition. }\end{array}$ & $\begin{array}{l}\text { OverStitch, Apollo Endo-surgery; Method: flexible } \\
\text { endoscopic suturing system; Follow-up: } 6 \mathrm{~m} \text {. }\end{array}$ & $\begin{array}{l}\text { Quality of Life (6m follow-up): GIQLI scores: Baseline: } 105 . \\
\text { Follow-up: 119. Calculated change: +14 (range: 3-24). Data } \\
\text { reported graphically. }\end{array}$ \\
\hline $\begin{array}{l}\text { Fuller et al, } 2013{ }^{[42]} \text {; RCT-; } 2008-2010 ; \text { Australia; } \\
\text { Treatment: IGB: } \mathrm{n}=31,68 \% \mathrm{~F}, \mu 43 \mathrm{y}, 36 \mathrm{~kg} / \mathrm{m} 2 \text {. } \\
\text { Control: Lifestyle modification } \mathrm{n}=35,66 \% \mathrm{~F} \text {, } \\
\mu 48.1 \mathrm{y}, 36.7 \mathrm{~kg} / \mathrm{m}^{2}, 26 \% \text { attrition (ITT used) }\end{array}$ & $\begin{array}{l}\text { Orbera; Duration of Tx: } 12 \mathrm{~m} \text {; Method: inserted using } \\
\text { standard protocol [57], } 450-700 \mathrm{ml} \text { saline; Follow-up: } \\
6 \mathrm{~m} \text {, every } 3 \mathrm{~m} \text {. }\end{array}$ & $\begin{array}{l}\text { Quality of Life (6m follow-up): IWQOL-Lite: Baseline: } 60.7+16 \text {. } \\
\text { Calculated change: } 20.9 \text {. }\end{array}$ \\
\hline $\begin{array}{l}\text { Alfredo et al, } 2014{ }^{[41]} \text {; Prospective study; Italy; } \\
\text { IGB: } n=83,77 \% \mathrm{~F}, \mu 37.4 \mathrm{y}, \mathrm{BMI} 43.74 \mathrm{~kg} / \mathrm{m}^{2}, 41 \% \\
\text { attrition. }\end{array}$ & $\begin{array}{l}\text { BIB; Duration of Tx: } 6 \mathrm{~m} \text {; Method: Propofol sedation, } \\
500 \mathrm{~mL} \text { saline. Multiple IGB: Reintroduced after weight } \\
\text { gain } \geq 50 \%, \mathrm{n}=83 \text { had } 2 \text { nd IGB, } n=22(18 \%) \text { had 3rd } \\
\text { IGB, } n=1(1.2 \%) \text { had } 4 \text { th IGB; Follow-up: } 12 \mathrm{~m}, 6 \mathrm{y} \text {. }\end{array}$ & $\begin{array}{l}\text { Quality of life (76m follow-up) } \mathbf{n}=\mathbf{6 4} / \mathbf{8 3}: \mathrm{SF}-12 \text { : Baseline: } \\
\text { Physical: } \mu 40+\mathrm{SE}(4), \text { Mental health: } \mu 50+\mathrm{SE}(4) \text {. Follow-up: } \\
\text { Physical: } \mu 85+\mathrm{SE}(8) \text {, calculated change: }+45 \text {, Mental health: } \mu 78 \\
+ \text { SE (7), calculated change: }+28, \mathrm{p}=<0.001 \text {. Data reported } \\
\text { graphically. }\end{array}$ \\
\hline
\end{tabular}


Guedes et al, 2019. ${ }^{[0]}$; Prospective study-; 2016-
2018; Brazil; IGB: $\mathrm{n}=42 ; 76 \% \mathrm{~F}, \mu 37.60 \pm 1.28 \mathrm{y}$, BMI $\mu 35.15 \pm 0.41 \mathrm{~kg} / \mathrm{m}^{2}, 0 \%$ attrition.

Marinos et al, 2014. ${ }^{[49]}$; Prospective study; Australia; Transpyloric shuttle $3 \mathrm{~m}: \mathrm{n}=10,90 \% \mathrm{~F}$, $\mu 36.3+11.4 \mathrm{y}, \mathrm{BMI} \mu 34+1.3 \mathrm{~kg} / \mathrm{m} 2$. Transpyloric Shuttle $6 \mathrm{~m}: \mathrm{n}=10,90 \% \mathrm{~F}, \mu 45+8.3 \mathrm{y}$, BMI $\mu 37.9+7.3 \mathrm{~kg} / \mathrm{m}^{2}, 0 \%$ attrition.

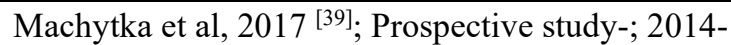
2015; Czech Republic \& Greece; IGB: $n=34 ; 67 \% \mathrm{~F}$, $42+11$ y (range: $18-59 y$ ), BMI $34.8+3.7 \mathrm{~kg} / \mathrm{m} 2$ (range: $27-40 \mathrm{~kg} / \mathrm{m}^{2}$ ), $6 \%$ attrition.

Moreno et al, $2008^{[48]}$; Prospective study; Belgium; TOGA: $\mathrm{n}=11,64 \% \mathrm{~F}, \mu 44.2+10.7 \mathrm{y}$, BMI $\mu 41.6+4.3 \mathrm{~kg} / \mathrm{m}^{2}, 0 \%$ attrition.

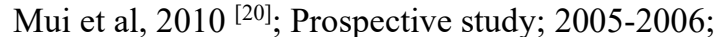
China; IGB: $\mathrm{n}=119,72 \% \mathrm{~F}, \mu 37.8+10 \mathrm{y}, \mathrm{BMI}$ : $\mu 38.4+8.0 \mathrm{~kg} / \mathrm{m} 2$, (range: $26.5-69.1 \mathrm{~kg} / \mathrm{m}^{2}$ ), $0 \%$ attrition.

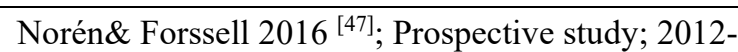
2013; Sweden; Aspiration Therapy: $\mathrm{n}=25,92 \% \mathrm{~F}$, $\mu 48 \mathrm{y}$, (range: $33-65 \mathrm{y}$ ), BMI $\mu 39.8+4.3 \mathrm{~kg} / \mathrm{m}^{2}, 20 \%$ attrition.

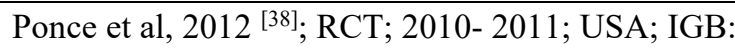
$\mathrm{n}=21,81 \% \mathrm{~F}, \mu 38.9+9.1 \mathrm{y}, \mathrm{BMI} \mu 34.7+2.6 \mathrm{~kg} / \mathrm{m}^{2}, 5 \%$ attrition.

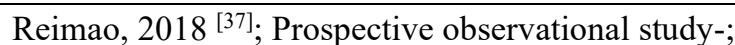
2014-2016; Brazil; IGB: $\mathrm{n}=40,78 \% \mathrm{~F}, \mu 45.3+7.6 \mathrm{y}$ (range: $25-57 \mathrm{y}$ ), BMI $\mu 32.9+2.0 \mathrm{~kg} / \mathrm{m}^{2}, 10 \%$ attrition.
Orbera or Spatz; Duration of Tx: 6m; Method: inserted under sedation, $600-700 \mathrm{~mL}$ saline, containing $4 \%$ methylene blue; Follow-up: $6 \mathrm{~m}$, monthly.

TransPyloric Shuttle; Duration of Tx: 3m; Method: inserted under sedation; Follow-up: $6 \mathrm{~m}$

TransPyloric Shuttle; Duration of Tx: 6m; Follow-up: $6 \mathrm{~m}$

Elipse device; Duration of Tx: 4m (range: standard 117 141d; experimental 30-141); Method: IGB inside capsule, attached to catheter via a patented self-sealing valve and swallowed, $550 \mathrm{~mL}$ of fluid $(\mathrm{n}=28)$; Followup: fortnightly.

TOGA System sleeve stapler; Method: inserted under sedation; Follow-up: monthly.

BioEnterics IGB; Duration of Tx: 6m; Method: inserted $\&$ removed by surgical team, $\mu 542.7+28.2 \mathrm{~mL}$; Followup: weekly with dietitian for $1 \mathrm{~m}$, then monthly.

Aspire Assist System; Duration of Tx: 12m (optional additional $12 \mathrm{~m}$ ); Method: custom gastrostomy tube percutaneously inserted during gastroscopy under sedation. Drainage \& irrigation of stomach $3 x /$ day Follow-up: 4 in $3 \mathrm{~m}$, then every $3 \mathrm{~m}$.

ReShape Duo IGB System; Duration of Tx: 6m;

Method: $900 \mathrm{~mL}$ saline; Follow-up: monthly-6m, biweekly-48weeks.

Orberra IGB; Duration of Tx: $6 \mathrm{~m}$; Method: inserted under general anaesthesia, $600 \mathrm{~mL}$ saline and methylene blue dye; Follow-up: monthly (nutritionist).
Quality of Life (6m follow-up): SF-36 mean + IQR score: Baseline: general health: (72, 57-82), MH (52, 40-72). Follow-up: general health $(82,72-92, \mathrm{p}=0.0002)$, $\mathrm{MH}(76,68-84, \mathrm{p}=0.0003)$. Calculated change: general health: $+10, \mathrm{MH}:+24$.

Quality of Life (6m follow-up):

IWQOL-Lite score: Calculated change: $+20.4+14.2$.

Quality of Life (6m follow-up): IWQOL-Lite score: Calculated change: $+23.3+20.5$.

Quality of Life (4m follow-up): IWQoL total score $(\mathrm{n}=26)$ : Baseline: $\mu 68$. Follow-up $\mu 82 \pm 12.2$. Calculated change: -14 .

Quality of Life (6m follow-up): SF-36 total score: Baseline: 96 Follow-up: 49.9. Calculated change: -46; IWQOL-Lite domain scores: general health (40.4), MH (40). Follow-up: general health (56.7), $\mathrm{MH}$ (50). Calculated change: general health: $+16.3, \mathrm{MH}$ : $+10$

Quality of life (6m follow-up): SF-36: (Chinese Version): General health baseline: $\mu 40.3+10.5$, follow-up: $49.4+11.3$, calculated change: $\mu-9.1$. MH: baseline: $\mu 43.8+12.4$, follow-up: $\mu 47.7+13.3$, calculated change: $\mu 3.9,(\mathrm{p}>0.014)$.

Quality of life (12m follow-up): EQ-5D baseline: $\mu 0.7 \pm 0.3$, follow-up: $\mu 0.9+0.1(\mathrm{p}<0.01)$, VAS baseline: $\mu 63+15$, follow-up: $\mu 83+14,(\mathrm{p}<0.01)$

Quality of Life (6m follow-up): SF-36 domain scores: baseline general health (73.9), $\mathrm{MH}$ (87.3). Follow-up: general health (80.7), $\mathrm{MH}$ (86.1). Calculated change: general health $+6.8, \mathrm{MH}-1.2$. Quality of life (6m follow-up): SF-36 (validated Portuguese version) General Health (\%) baseline: $\mu 43$, follow-up: $\mu 68$ calculated change: +25 . MH (\%) baseline: $\mu 62$, follow-up: $\mu 79$, calculated change: +17 . Data reported graphically. 
Raftopoulos et al, 2017. ${ }^{[36]}$; Prospective observational, nonrandomised study; Greece; IGB: $\mathrm{n}=12,58 \% \mathrm{~F}, \mu 41 \mathrm{y}$ (range: $18-59 \mathrm{y}$ ), BMI $\mu 36.1+3.2 \mathrm{~kg} / \mathrm{m}^{2}, 8 \%$ attrition

Guedes et al 2017 ${ }^{[2]}$, Guedes et al $2016^{[35]}$; Prospective observational study-; 2011-2012; Brazil IGB: $\mathrm{n}=50,80 \% \mathrm{~F}, \mu 34.6+7.1 \mathrm{y}, \mathrm{BMI} \mu 40+6.3$ $\mathrm{kg} / \mathrm{m}^{2}, 22 \%$ attrition.
Elipse Balloon; Duration of Tx: 4m; Method: insertion via swallow with water, $550 \mathrm{~mL}$ water containing citric acid/potassium sorbate preservative; Follow-up: fortnightly.

Silmed Silicone IGB; Duration of Tx: 6m; Method: inserted under sedation, $650 \mathrm{~mL}$ saline solution $(0.9 \%)$ and $20 \mathrm{~mL}$ methylene blue solution; Follow-up: weeks $0,8,16$ \& 24

Quality of Life (12m follow-up): IWQOL-Lite score: Baseline 65. Follow-up: 58. Calculated change: -7 .

Quality of life (6m follow-up): WHOQOL-BREF Physical domain baseline; $\mu 54.3 \pm 17.9$, follow-up; $\mu 67.0 \pm 16.2, \mathrm{p}<0.01$, Psychological domain baseline; $\mu 55.9+17.2$, follow-up; $\mu 64.5+19.9, \mathrm{p}$

0.03 . Calculated change: physical: +12.7 , psychological: +8.6 .

Depression/Anxiety (6m follow-up): *BDI: Baseline: $\mu 16$ (median), (range: 1-32), follow-up; $\mu 6$, (range: 0-45), change: $\mu 4.57 \pm 10.6,(p=0.0019)$; HADS-D baseline: $\mu 7$ (range: $1-14)$, follow-up: $4(0-18)$, change: $1.82+5.16,(\mathrm{p}=0.0345)$.

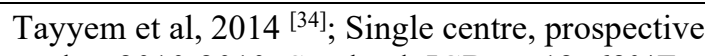
study-; 2010-2010; Scotland; IGB: $n=12,62 \% \mathrm{~F}$, $\mu 40 \mathrm{y}$, BMI $\mu 55.9 \mathrm{~kg} / \mathrm{m}^{2}$, attrition unclear. Tayyem et al, $2011^{\text {[33] }}$; Prospective study; 20082010; IGB: $\mathrm{n}=17,65 \% \mathrm{~F}, \mu 40.9 \mathrm{y}, \mathrm{BMI} \mu 61.4+8.3$ $\mathrm{kg} / \mathrm{m}^{2}, 0 \%$ attrition.

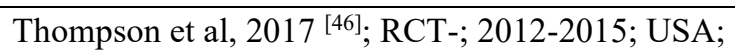
Aspiration therapy: $\mathrm{n}=111,83 \% \mathrm{~F}, \mu 43.5+10.2 \mathrm{y}$, BMI $\mu 42.4+5.0 \mathrm{~kg} / \mathrm{m}^{2}, 26 \%$ attrition.

BioEnterics IGB (BIB) System; Duration of Tx: 6m; Method: inserted under sedation, $600 \mathrm{~mL}$ saline containing methylene blue.

BioEnterics IGB (BIB) System; Duration of Tx: 6m;

Method: inserted under sedation, $600 \mathrm{~mL}$ saline containing methylene blue; Follow-up: quarterly.
Quality of Life: SF-36 domain scores: Baseline general health: 29. Follow-up: general health: 63. Calculated change: general health: +34 .

Quality of Life (9m follow-up): SF-36 domain scores: Baseline general health: 28. Follow-up: general health: 70. Calculated change: general health: $+42, p<0.021$. Data reported graphically.

Aspire Assist System; Duration of Tx: 52w; Method: Endoscopically placed percutaneous gastrostomy tube, external device for drainage 20mins post-meal; FollowQuality of life (12m follow-up): IWQOL Total Score change: $\mu 6.2+13.4$.

up: week $0,2,6,10,14,20,24,28,32,36,40,44,48 \& 52$.

BDI: Beck Depression Inventory; BDI-II; Beck Depression Inventory II; BFM: Body fat mass; BMI: body mass index; BW: Body weight; CRP: C-reactive Protein; DBP:

Diastolic blood pressure; DLD: dyslipidaemia; DM: diabetes mellitus; ED: Eating disorder; EQ-5D: European Quality of life measurement questionnaire; ESL: English as a second language; EW: Excess weight; EWL: excess weight loss; FBGL: Fasting blood glucose; GIQLI: Gastrointestinal Quality of Life Index; Hx: history; HDAS-A: Hospital Anxiety and Depression Scale (Anxiety score); HDAS-D: Hospital Anxiety and Depression Scale (Depression Score); HTN: hypertension; IBD: inflammatory bowel disease; IGB: intragastric balloon; IQR: interquartile range; ITT, intention to treat; IWQOL-Lite: Impact of Weight on QOL-Lite; LDL: low density lipoprotein; MH: mental health; MI: myocardial infarction; MS: Metabolic Syndrome; NR: not reported; QOL: quality of life; SBP: Systolic blood pressure; SD: standard deviation; SF-12: Quality Metric's Short Form; SF-36: 36-Item Short-Form Health Survey; TBWL: Total body weight loss; TC: Total Cholesterol; TG: Triglycerides; TOGA: transoral gastroplasty; VAS: Visual Analogue Scale; WC: Waist circumference; WL: Weight loss.

BDI: score decreases ${ }^{[58]}$

BDI-II: score decreases ${ }^{[59]}$

EQ-5D: score increases ${ }^{[60]}$

GIQLI: score increases. 4 is the most desirable option, 0 is the least desirable option ${ }^{[61]}$

HDAS: score decreases ${ }^{[62]}$ IWQOL-BREF: A higher score indicates an improved quality of life ${ }^{[63]}$ 
IWQOL-Lite: score increases. Scores range from 0 to 100 , with 100 representing the best quality of life [64]

IWQOL: higher scores indicated lower levels of functioning and QOL ${ }^{[65]}$

SF-12: score decreases ${ }^{[66]}$

SF-36: A higher score indicates a better health status ${ }^{[67]}$ 


\section{Table S1: Systematic search strategy}

MEDLINE (via PubMed) was searched 21st October 2019 using keywords (title and abstract) and MeSH Terms. Result $=1421$ records

(((("Obesity, Morbid /surgery"[Mesh] OR ("morbid obesity"[tiab] AND surgery[tiab])) OR "Obesity, Morbid /therapy"[Mesh]) OR ("Morbid obesity"[tiab] AND therapy[tiab]))) OR $(((()((()((()(()(()((($ Gastroplasty[Mesh]) OR Gastroplasty[tiab]) OR "Single-Balloon Enteroscopy"[Mesh]) OR "Single-balloon enteroscopy"[tiab]) OR "Double-Balloon Enteroscopy"[Mesh]) OR "Double-balloon enteroscopy"[tiab]) OR "Double-balloon enteroscopy"[tiab]) OR Bioenterics[tiab]) OR Orbera[tiab]) OR Spatz[tiab]) OR "transpyloric shuttle"[tiab]) OR "endoscopic sleeve gastroplasty"[tiab]) OR "aspire assist"[tiab]) OR ((incisionless[tiab]) AND "anastomosis, surgical"[Mesh])) OR "anastomotic system"[tiab]) OR "Balloon Enteroscopy"[Mesh]) OR "balloon enteroscopy"[tiab]) OR "Gastric balloon"[tiab]) OR "intragastric balloon*"[tiab]) OR BIB[tiab]) OR IB[tiab]) OR "ESG bariatric"[tiab]) OR "gastric bubble"[tiab]) OR "intragastric bubble"[tiab])AND (((("Quality of life"[Mesh]) OR "quality of life"[tiab]) OR hrql[tiab]) OR qol[tiab]) OR hrqol[tiab])

CINAHL (via Ebscohost) was searched on 21 st October 2019 using keywords and CINAHL Headings. Results $=109$ records

TI (hrqol) OR AB (hrqol) OR TI (hrql) OR AB (hrql) OR TI ("Quality of Life") OR AB ("Quality of Life") OR MH "Quality of Life" AND TI ("gastric bubble") OR AB ("gastric bubble") OR TI (BIB) OR AB (BIB) OR TI ("double-balloon enteroscopy") OR AB ("double-balloon enteroscopy") OR TI ("Single-balloon enteroscopy") OR AB ("Single-balloon enteroscopy") OR TI ("intragastric balloon") OR AB ("intrgastric balloon") OR TI ("gastric balloon") OR AB ("gastric balloon") OR TI ("aspire assist") OR AB ("aspire assist") OR TI ("endoscopic sleeve gastroplasty") OR AB ("endoscopic sleeve gastroplasty") OR TI ("transpyloric shuttle") OR AB ("transpyloric shuttle") OR TI (spatz) OR AB (spatz) OR TI (orbera) OR AB (orbera) OR TI (Bioenterics) OR AB (Bioenterics) OR TI ("balloon enteroscopy") OR AB ("balloon enteroscopy") OR MH "Balloon Enteroscopy" OR TI (gastroplasty) OR AB (gastroplasty) OR MH "Gastroplasty" AND ( TI ("morbid obesity") OR AB ("morbid obesity")) OR (TI (surgery) OR AB (surgery)) OR MH "Obesity, Morbid" AND (TI ("therapy") OR AB ("therapy"))

EMBASE was searched 21st October 2019 for citations from both Embase and MEDLINE using keywords (abstract and title) and Emtree terms Results = 4066 records

hrqol:ab,ti OR hrql:ab,ti OR 'quality of life'/exp OR 'quality of life':ab,ti AND bib:ab,ti OR 'gastric bubble':ti,ab OR 'esg bariatric':ti,ab OR ib:ti,ab OR 'stomach bypass device':ti,ab OR 'intragastric balloon':ti,ab OR 'gastric balloon'/exp OR 'gastric balloon':ti,ab OR 'balloon enteroscopy':ti,ab OR 'balloon enteroscopy'/exp OR 'aspire assist':ti,ab OR 'transpyloric shuttle':ti,ab OR orberra:ti,ab OR spatz:ti,ab OR bioenterics.ti,ab OR 'double balloon enteroscopy':ti,ab OR 'single balloon enteroscopy':ti,ab OR 'single balloon enteroscopy'/exp OR 'endoscopic sleeve gastroplasty'/exp OR 'endoscopic sleeve gastroplasty':ti,ab OR 'obesity therapy':ti,ab OR 'obesity therapy'/exp OR 'stomach bypass device'/exp OR 'morbid obesity':ab,ti OR 'surgery'/exp OR 'bariatric surgery'/exp OR 'anastomotic system':ti,ab OR 'anastomosis, surgical':ti,ab OR 'anastomosis and surgical':ti,ab OR incisionless:ti,ab

PsycINFO was searched 22nd October 2019 using keywords (title and abstract) and PsycINFO Terms. Result $=362$ records 
exp Obesity/ and exp Surgery/ OR "morbid obesity".ab,ti. OR surgery.ab,ti. OR therapy.ab,ti OR gastroplasty.ab,ti. OR "Single-Balloon Enteroscopy".ab,ti. OR "Double-Balloon Enteroscopy".ab,ti. OR Bioenterics.ab,ti. OR Orbera.ab,ti. OR Spatz.ab,ti. OR "transpyloric shuttle".ab,ti. OR "endoscopic sleeve gastroplasty".ab,ti. OR "aspire assist".ab,ti. OR incisionless.ab,ti. OR "anastomosis, surgical".ab,ti. OR "anastomotic system".ab,ti. OR "Balloon Enteroscopy".ab,ti. OR "Gastric balloon".ab,ti. OR "intragastric balloon".ab,ti. OR BIB.ab,ti. OR IB.ab,ti. OR "ESG bariatric".ab,ti. OR "gastric bubble".ab,ti. OR "intragastric bubble".ab,ti. AND exp *"Quality of Life"/ OR hrql.ab,ti. OR hrqol.ab,ti. OR qol.ab,ti.

Total

5958 records 
Table S2: Extended version of characteristics and outcomes of the 20 included publications which reported quality of life and/or mental health pre and post endoscopy bariatric therapies in adults.

\begin{tabular}{|c|c|c|c|}
\hline $\begin{array}{l}\text { Study design, setting and } \\
\text { participants }\end{array}$ & Endoscopic therapy & Outcomes & Comment \\
\hline $\begin{array}{l}\text { Ahmed et al } 2019 \text { (56) } \\
\text { Prospective } 2 \text {-arm ( } 1 \text { =endoscopic; } \\
\text { 1=Atkins diet) randomised } \\
\text { descriptive longitudinal study-; 2008- } \\
12 \text {. } \\
\text { Exclusion: psychological problems, } \\
\text { taking psychotropic drugs, previous } \\
\text { IB or bariatric surgery, peptic ulcers, } \\
\text { binge eating disorders. } \\
\text { Iraq } \\
\text { BIB: } n=40,100 \% F, \mu 27 y \text { (range: } 20- \\
39 y), \text { BMI } \mu 36 \text { (range: } 31-39.9) \\
\mathrm{kg} / \mathrm{m} 2 \text {. }\end{array}$ & $\begin{array}{l}\text { BIB } \\
\text { Duration of Tx: } 6 \mathrm{~m} \\
\text { Method: inserted under } \\
\text { sedation, } 600 \mathrm{~mL} \text { saline containing } 10 \% \\
\text { methyl blue. } \\
\text { Follow-up: } \mathrm{d} 7, \mathrm{~d} 14 \text {, then monthly. }\end{array}$ & $\begin{array}{l}\text { Quality of life (6m follow-up): } \\
\text { EQ-5D: NR. } \\
\text { Weight loss: } \\
\text { EWL\%: } 31-35 \mathrm{~kg}(47.5 \%), \mathrm{p}=0.00001\end{array}$ & $\begin{array}{l}\text { No funding received. } \\
\text { Author contacted about type of } \\
\text { QOL tool used; could not } \\
\text { provide data. } \\
\text { Participants reported daily } \\
\text { teasing prior to therapy, afraid } \\
\text { of media stating risks of } \\
\text { obesity, obesity made } \\
\text { participants uneasy socialising } \\
\text { with friends - narrowed social } \\
\text { circles and weight as an } \\
\text { obstacle to obtaining a job. }\end{array}$ \\
\hline \multirow{2}{*}{$\begin{array}{l}\text { De Castro et al } 2010 \text { (55) } \\
\text { Prospective } 2 \text {-arm }(2=\text { endoscopic }) \\
\text { double-blinded study; 2006-9. } \\
\text { Exclusion: disease of upper GIT, } \\
\text { hiatus hernia }>3 \mathrm{~cm} \text {, anti- } \\
\text { inflammatory agents, } \\
\text { anticoagulants. } \\
\text { Spain. } \\
\text { BIB: } \mathrm{n}=15,67 \% \mathrm{~F}, \mu 45.4 \pm 8 \mathrm{y}, \mathrm{BMI} \\
\mu 44.2 \pm 6 \mathrm{~kg} / \mathrm{m} 2 \text {. } \\
\text { Heliosphere IGB: } \mathrm{n}=18,72 \% \mathrm{~F}, \\
\mu 42.7 \pm 12 \mathrm{y}, \text { BMI } \mu 44.2 \pm 5 \mathrm{~kg} / \mathrm{m} 2 .\end{array}$} & $\begin{array}{l}\text { BIB } \\
\text { Duration of Tx: } 6 \mathrm{~m} \\
\text { Method: inserted under conscious } \\
\text { sedation, } 700 \mathrm{ml} \text { saline containing } \\
\text { methylene blue. } \\
\text { Follow-up: monthly. }\end{array}$ & $\begin{array}{l}\text { Quality of life (6m follow-up): } \\
\text { GIQLI score: baseline } \mu 86.9 \pm 17 \text {. Follow-up: } \mu 83.6 \pm 12 \text {. } \\
\text { Calculated change: }-3.3 \text {. } \\
\text { Weight loss: } \\
\text { Baseline: } \mu 121+18 \mathrm{~kg} \text {. Calculated change: }-13 \mathrm{~kg} \text {. } \\
\text { EWL\%: } \mu 30.2 \pm 19 \% \text {. } \\
\quad \text { Adverse events: } \\
\mathrm{n}=3 / 15 \text { continuous vomiting and dehydration. }\end{array}$ & Funded by a FISS grant. \\
\hline & $\begin{array}{l}\text { Heliosphere IGB } \\
\text { Duration of Tx: } 6 \mathrm{~m} \\
\text { Method: } 960 \mathrm{~cm} 3 \text { air } \\
\text { Follow-up: monthly. }\end{array}$ & $\begin{array}{l}\text { Quality of life ( } 6 \mathrm{~m} \text { follow-up): } \\
\text { GIGLI score: baseline } \mu 92.2+18 \text {. Follow-up: } \mu 102.4 \pm 23 \text {. } \\
\text { Calculated change: }+10.2 \text {. } \\
\text { Weight loss: } \\
\text { Baseline: } \mu 119+17 \mathrm{~kg} \text {. Calculated change: }-13 \mathrm{~kg} \text {. } \\
\text { EWL\%: } \mu 27 \pm 16 \text {. }\end{array}$ & \\
\hline $\begin{array}{l}\text { Deliopoulo et al } 2013(54) \\
\text { Prospective study-; } 2009-2010 . \\
\text { Exclusion: no alcohol, drug problems } \\
\text { or active psychosis. } \\
\text { Greece } \\
\text { IGB: } \mathrm{n}=100\end{array}$ & $\begin{array}{l}\text { BioEnterics IGB } \\
\text { Duration of Tx: } 6 \mathrm{~m} \\
\text { Method: inserted under conscious or } \\
\text { unconscious sedation under endoscopic } \\
\text { vision. }\end{array}$ & $\begin{array}{l}\text { Depression symptoms }(6 \mathrm{~m} \text { follow-up): } \\
\text { BDI-II baseline: } \mu 20.3+8.5 \text { [range: } 10-54] . \text { Follow-up: } \mu 7.9+5.6 \\
\text { [range: } 0-26], \mathrm{p}<0.0001 . \text { Calculated change }=-12.4 . \\
\text { Weight loss }(\mathrm{kg}): \\
\text { Baseline: } \mu 124.7+32.3 \mathrm{~kg} \text {. Follow up: } \mu 103.7+30.1 \mathrm{~kg}, \mathrm{p}=0.983 \text {. } \\
\text { Calculated change: }-21 \mathrm{~kg} \text {. }\end{array}$ & $\begin{array}{l}\text { No funding received. } \\
\text { Authors contacted about brand } \\
\text { of balloon \& funding source. } \\
\text { Theorised self-esteem and } \\
\text { subjective well-being are } \\
\text { influenced by poor self- }\end{array}$ \\
\hline
\end{tabular}


Depressed group: $\mathrm{n}=65$ (mild-26, moderate- 21 , severe- 18 ), $100 \% \mathrm{~F}$,

$\mu 37.52+11.77 y$ [median: 37, range: $19-61], \mu 43.5+9.5 \mathrm{~kg} / \mathrm{m} 2$.

Non-depressed group: $\mathrm{n}=35,100 \% \mathrm{~F}$ $33.89+11.50 y$ [33,18-63], BMI 41.9 $+7.4 \mathrm{~kg} / \mathrm{m} 2$.

Familiari et al. 2011. (13)

Prospective single-arm study-; 20072010.

Exclusion: $\mathrm{BMI}>55 \mathrm{~kg} / \mathrm{m} 2$, hiatus hernia $>2 \mathrm{~cm}$, previous bariatric surgery, inflammatory disease of

GIT, pregnancy or breast feeding,

HIV, esophagitis, alcohol/drug

addiction, present infection, thyroid

disease, hx of scleroderma.

Italy \& Belgium.

TOGA: $n=67$; follow-up $n=53$;

$70 \% \mathrm{~F}, \mu 41.0+9.7 \mathrm{y}$,

BMI $\mu 41.5+3.6 \mathrm{~kg} / \mathrm{m} 2$.

Fiorillo et al. 2020 (14)

Retrospective single-centre study-

2016-2018.

France

ESG: $n=42$; follow-up $n=23 ; 16 \mathrm{~F}$

(69.6\%), $\mu 41 \mathrm{y}$ (range: $35-43 \mathrm{y}), \mathrm{BMI}$

39.5 (range: $36.7-44.7$ ) kg/m2 .

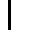

\section{Fuller et al 2013 (53)}

RCT-; 2008-2010.

Exclusion: Conditions increasing the

risks associated with endoscopy or protocol (61), 450-700ml saline.

insertion of IGB, inflammation of Follow-up: $6 \mathrm{~m}$, every $3 \mathrm{~m}$.

VerStitch, Apollo Endo-surgery.

OGA sleeve stapler $\&$ restrictor

ystems.

tapler device.

Method: flexible endoscopic suturing

system.

Follow-up: 6m.
Follow-up: monthly.

EWL $\%: \mu 39.6 \%$.

Weight loss $(\mathrm{kg})$

Baseline: $\mu 122.3+24.2 \mathrm{~kg}$. Follow up: $\mu 103.6+24.1 \mathrm{~kg}$.

Calculated change $=-18.7 \mathrm{~kg}$.

EWL\%: $\mu 36.1 \mathrm{~kg}$.

Quality of Life (12m follow-up):

SF-36v2 \& IWQOL-Lite: $\mathrm{p}=<0.001$.

Weight loss:

Baseline: $\mu 116.6+18.5 \mathrm{~kg}$. Calculated change: $-19+8.5 \mathrm{~kg}$.

EWL\%: $\mu 38.7+7.1 \%$.

Comorbidities:

Baseline: DM $\mathrm{n}=4 / 67$

Follow-up: DM n=3/53 ( $\mathrm{p}=0.0005)$.

$2 \mathrm{~m}$ behavioural modification program

\section{QOL (6m follow-up):}

GIQLI scores: Baseline: 105. Follow-up: 119. Calculated change: +14 (range: $3-24$ ).

Data reported graphically.

Weight loss:

Baseline: $\mu 115.5+29.6 \mathrm{~kg}$

EWL\%: 39.9 (range: 17.5-58.9) \%.

Comorbidities:

Baseline: DM: $n=2 / 23$, HTN $n=3 / 23$, OSA $n=5 / 23$. Follow-up: DM $n=1 / 23$, HTN $n=2 / 23$, OSA $n=2 / 23$.

QOL (6m follow-up):

IWQOL-Lite: Baseline: $60.7+16$. Calculated change: 20.9 .

Weight loss:

Baseline: $\mu 104.6 \mathrm{~kg}$. Calculated change: $-14.4 \mathrm{~kg}$

$\%$ EWL: $\mu 50.3 \%$

hx of symptoms of oesophageal or GI(diet and exercise).

reported physical health and body image" than body weight itself.

Funding by Satiety Inc.

$\mathrm{n}=2$ underwent laparoscopic bariatric procedures within

$12 \mathrm{~m}$-post TOGA. 
motility disorders, hiatus hernia

$>5 \mathrm{~cm}$, structural abnormality of the Control Group

GI tract, prior gastric surgery or IGB, T2DM Lifestyle Intervention

or major surgery within $3 \mathrm{~m}$, Program.

cerebrovascular or cardiopulmonary 12 months, 3 months

disease, uncontrolled BP

(>160/95 mmHG), epilepsy, T1DM,

undiagnosed thyroid disease or

hypothyroidism in which the dose of

thyroxine replacement has not been

stable for at least $3 \mathrm{~m}$, hepatic or renal

insufficiency, psychiatric disorder or

pregnancy.

Australia

Treatment: IGB: $\mathrm{n}=31,68 \% \mathrm{~F}, \mu 43 \mathrm{y}$,

$36 \mathrm{~kg} / \mathrm{m} 2$.

Control: Lifestyle modification $\mathrm{n}=35$

$66 \% \mathrm{~F}, \mu 48.1 \mathrm{y}, 36.7 \mathrm{~kg} / \mathrm{m} 2$.

Alfredo et al, 2014 (52)

Prospective 6y follow-up study.

Exclusion: Weight loss $>5 \%$ or

medication causing weight gain (e.g.

glucocorticoids or second generation

anti-psychotic medication).

Italy

IGB: $\mathrm{n}=83$, follow-up: $\mathrm{n}=49,64 \mathrm{~F}$

$(77 \%), \mu 37.4 y$, BMI $43.74 \mathrm{~kg} / \mathrm{m} 2$.
$75 \%$ nausea/vomiting, 39\% reflux, 33\% lethargy, 55\% abdominal pain/cramping in week 1.

Comorbidities:

Baseline: metabolic syndrome: $31 / 31$.

Follow-up: metabolic syndrome: $15 / 31$
BIB

Duration of Tx: $6 \mathrm{~m}$

by anaesthetist, $500 \mathrm{~mL}$ saline.

IGB: Reintroduced after

weight gain $>50 \%, n=83$ had 2 nd IGB,

$n=22(18 \%)$ had 3 rd IGB, $n=1(1.2 \%)$

had 4th IGB.

Follow-up: $12 \mathrm{~m}, 6 \mathrm{y}$

Low-calorie diet provided by dietitian.
Method: Propofol sedation administered

Weight loss (12m follow-up)

BMI: $35.9 \mathrm{~kg} / \mathrm{m} 2$, (change: $-7.8 \mathrm{~kg} / \mathrm{m} 2$ ), $\mathrm{p}=<0.001$.

Quality of life (76m follow-up) $n=64 / 83$

SF-12: Baseline: Physical: $\mu 40+$ SE (4), Mental health: $\mu 50+$ SE

(4). Follow-up: Physical: $\mu 85+\operatorname{SE}(8)$, calculated change: +45 ,

Mental health: $\mu 78+$ SE $(7)$, calculated change: $+28, p=<0.001$.

Data reported graphically.

Comorbidities:

Baseline: T2DM: 33/83, HTN: 58/83, OSA: 16/83, p=0.02.

Follow-up: T2DM: 14/49, HTN: 17/49, OSA: 5/49.

Data reported graphically.

Adverse Events:

1 st IGB placement: Nausea, vomiting and epigastric pain $\mu 2.5 \mathrm{~d}$,

2nd IGB placement: Nausea, vomiting \& epigastric pain $\mu 4 \mathrm{~d}$. No major complications, IGB removal: $\mathrm{n}=1$ for intolerance.

Guedes et al., 2019. (51)

Orbera or Spatz

Quality of Life (6m follow-up):

SF-36 mean + IQR score: Baseline: general health: $(72,57-82)$,

MH (52, 40-72), functional capacity $(60,40-85)$. Follow-up:

Method: inserted under sedation

by anesthesiologist, $600-700 \mathrm{~mL}$ saline. general health $(82,72-92, \mathrm{p}=0.0002)$, MH $(76,68-84, \mathrm{p}=0.0003)$,

\begin{tabular}{l|l}
$\begin{array}{l}\text { hypothyroidism, PCOS), AIDS, } \\
\text { inflammatory conditions, malignant }\end{array}$ & containing 4\% methylene blue. \\
Follow-up: 6m, monthly.
\end{tabular}

functional capacity $(90,85-95, \mathrm{p}=0.0001)$. Calculated change:

general health: $+10, \mathrm{MH}:+24$, functional capacity: +30 
diseases, autoimmune diseases, $\mathrm{CKD}$, Individualised low-calorie diet provided Weight loss:

$\mathrm{HF}$, hepatic failure disorders,

medications interfering with

weight.

Brazil

IGB: $\mathrm{n}=42 ; 0 \%$ attrition, $76 \% \mathrm{~F}$,

$\mu 37.60 \pm 1.28 \mathrm{y}, \mathrm{BMI}$

$\mu 35.15 \pm 0.41 \mathrm{~kg} / \mathrm{m} 2$

Marinos et al., 2014. (60)

by dietitian.

Baseline: $\mu 96 \pm 1.9 \mathrm{~kg}$. Follow-up: $80.6 \pm 2.0 \mathrm{~kg}$. Calculated change:

$-15.4+1.5 \mathrm{~kg}$

EWL\%: $56.04 \pm 4.90 \%, \mathrm{p}=<0.0001$

Comorbidities:

Prospective open-label study.

Baseline: HTN: $n=6 / 42$, dyslipidaemia: $n=32 / 42$.

Exclusion: positive helicobacter

Duration of Tx: $3 \mathrm{~m}$

Quality of Life (6m follow-up):

IWQOL-Lite score: Calculated change: $+20.4+14.2$.

unding by BAROnova Inc.

Weight loss:

pylori, insulin-dependent DM, active Follow-up: 6m

gastric ulcer.

Australia.

Transpyloric shuttle $3 \mathrm{~m}: \mathrm{n}=10$

$90 \% \mathrm{~F}, \mu 36.3+11.4 \mathrm{y}, \mathrm{BMI}$

TransPyloric Shuttle

Baseline: $\mu 98+8.1 \mathrm{~kg}$

EWL\%: $25.1+14 \%$

$\mu 34+1.3 \mathrm{~kg} / \mathrm{m} 2$.

Transpyloric Shuttle $6 \mathrm{~m}: \mathrm{n}=10$,

$90 \% \mathrm{~F}, \mu 45+8.3 \mathrm{y}, \mathrm{BMI}$

$\mu 37.9+7.3 \mathrm{~kg} / \mathrm{m} 2$.

\section{Machytka, E et al, 2017 (50)}

TransPyloric Shuttle

Duration of Tx: $6 \mathrm{~m}$

Quality of Life ( $6 \mathrm{~m}$ follow-up):

Follow-up: 6m

IWQOL-Lite score: Calculated change: $+23.3+20.5$.

Weight loss:

Baseline: $\mu 103.8+28.3 \mathrm{~kg}$

EWL\%: 41+21.1\%.

Adverse events:

Mucosal erosion: $n=15 / 20$, gastric ulcers: $n=10 / 20$.

Elipse device

Quality of Life (4m follow-up):

Prospective, observational and open Duration of Tx: 4m (range: standard

label design-; 2014-2015.

Exclusion: small bowel obstruction, Method: IGB folded inside capsule,

signs or symptoms of oesophageal,

gastric or intestinal disease, IBD,

IWQoL total score $(n=26)$ : Baseline: $\mu 68$. Follow-up $\mu 82+12.2$

Calculated change: -14 . Physical: baseline: $\mu 68+12.9$ follow-up

u 82 , calculated change: +14

attached to catheter via a patented self- Weight loss $(n=26)$ :

cancer or a known large hiatal hernia.fluid $(n=28)$. Experimental IGB made

More than 1 laparoscopic or

abdominal surgery and surgery in

$>12 \mathrm{~m}$, hx of smoking.

from radiopaque film, slightly smalle

capsules for ease of swallowing

$(\mathrm{n}=6)$.

Czech Republic \& Greece. $\quad$ Follow-up: fortnightly.

IGB: $n=34$; follow-up $n=32,23 F$

Nutritional counselling fortnightly \&

(67\%), 42+11y (range: $18-59 y$ ), BMI encouraged to follow a high protein

$34.8+3.7 \mathrm{~kg} / \mathrm{m} 2$ (range: 27 1000-1200 Calories/day diet.

$40 \mathrm{~kg} / \mathrm{m} 2)$.

BMI: Follow-up: $\mu-3.9+3.1 \mathrm{~kg} / \mathrm{m} 2(-5.2 \mathrm{CL},-2.6 \mathrm{CI}), \mathrm{p}<0.001$.

TBWL\%: follow-up $\mu 10+6.6 \%,(7.3,12.7), \mathrm{p}<0.001$

Adverse events $(\mathrm{n}=26)$ :

$n=24 / 26$, abdominal distension: $n=1 / 26$, abdominal pain: $n=7 / 26$, constipation: $n=5 / 26$, diarrhea: $n=4 / 26$, GERD: $n=3 / 26$, nausea:

$15 / 26$, vomiting: $n=18 / 26$.

Comorbidities (change at follow-up) $(n=26)$ :

HBA1c $(\mathrm{mg} / \mathrm{dL}): \mu-0.2+0.2 \%(-0.2,-0.009)$, LDL: $-\mu 9.7+27.6(-$

21.4,2.0), TG: $\mu-16.4+50.9(-37.9,5.1)$, SBP: $\mu-9.6+16.1(-16.2$, 2.9), DBP: $\mu-5.8+7.9(-9.0,-2.5)$

\begin{tabular}{lll}
\hline Moreno et al., 2008(59) & TOGA System sleeve stapler. & Quality of Life (6m follow-up)
\end{tabular}

Prospective single-arm study.

Funding by Satiety Inc.

Two authors received

consulting fees

from Allurion Technologies, 1

author is a consultant and 3

uthors are shareholders in the

company

Method: inserted under sedation. 
Exclusion: hx of IBD, pregnancy, cancer, etc.

Belgium

TOGA: $\mathrm{n}=11,64 \% \mathrm{~F}, \mu 44.2+10.7 \mathrm{y}$

BMI $\mu 41.6+4.3 \mathrm{~kg} / \mathrm{m} 2$.
Follow-up: monthly.

Diet and exercise guideline booklet

provided at follow-up

SF-36 total score: Baseline: 96. Follow-up: 49.9. Calculated

change: -46 .

IWQOL-Lite domain scores: physical function (38.9), general

health (40.4), MH (40). Follow-up: physical functioning (54.7),

general health (56.7), $\mathrm{MH}$ (50). Calculated change: physical

functioning: +15.8 , general health: $+16.3, \mathrm{MH}:+10$.

Weight loss:

Baseline: $\mu 119.8+22.2 \mathrm{~kg}$. Calculated change: $-24 \mathrm{~kg}$

EWL\%: $46 \%, p=<0.05$.

Adverse events:

Epigastric pain: $n=11 / 11$, esophagitis: $n=2 / 11$, throat pain: $n=3 / 11$,

nausea: $n=2 / 11$, mild dysphagia: $n=3 / 11$.

Comorbidities:

Baseline: T2DM: $n=4 / 11$ HTN $n=6 / 11$ hyperlipidaemia: $4 / 11$.

Mui et al 2010(22)

BioEnterics IGB

Quality of life (6m follow-up)

SF-36: (Chinese Version): Physical functioning baseline:

$\mu 28.8+19$, follow-up: $\mu 39.8+15.2$, calculated change: -11 ,

( $>0.0005)$. General health baseline: $\mu 40.3+10.5$, follow-up:

49.4+11.3, calculated change: $\mu-9.1$. MH: baseline: $\mu 43.8+12.4$,

follow-up: $\mu 47.7+13.3$, calculated change: $\mu 3.9,(p>0.014)$

Weight loss $(\mathrm{kg})$

Baseline: $\mu 103.7+24.1 \mathrm{~kg}$, (range: $63.8-183.6 \mathrm{~kg}$ ). Follow-up: $\mu$

$91.3 \pm 23 \mathrm{~kg}$. Calculated change: $-\mu 12.4+6.9 \mathrm{~kg}, \mathrm{p}<0.0005$.

EWL \%: $\mu 45.1 \pm 35.3 \%$.

Adverse events:

Intolerance (early removal): $n=4 / 119$, anaemia: $n=1 / 119$,

hypokalaemia: $\mathrm{n}=1 / 119$.

Comorbidities:

MS Baseline; $\mu$ 42.9\%, follow-up: $\mu 15.1 \%$,

FBG $(\mathrm{mmol} / \mathrm{l})$ baseline: $\mu 6.1+2.0$ follow-up $\mu 5.3+1.7$,

HBA1c $(\%)$ baseline: $\mu 7.4+1.6$, follow-up: $\mu 5.8+0.7,(\mathrm{p}<0.0005)$

TC $(\mathrm{mmol} / \mathrm{l})$ baseline; $\mu 5.1+0.9$, follow-up; $\mu 4.7+0.9(\mathrm{p}<0.0005)$,

TG $(\mathrm{mmol} / \mathrm{l})$ baseline; $\mu 1.7+1.0$ follow-up $\mu 1.3+0.7, \quad(\mathrm{p}<0.0005)$

SBP $(\mathrm{mmHg})$ baseline; $\mu 145.4+19.7$ follow-up;

$133.2+20.9(\mathrm{p}<0.005)$

DBP baseline; $\mu 84.3+12.6$, follow-up; $\mu 78.8+15.4(\mathrm{p}<0.005)$,

CRP $(\mathrm{mg} / \mathrm{l})$ baseline; $\mu 6.9 \pm 6$, follow-up; $\mu 6.1+6.5(\mathrm{p}<0.024)$ 
Norén, E.; Forssell, H, 2016 (58) Prospective observational study-; 2012-2013.

Sweden

Exclusion: $\mathrm{MI}<3 \mathrm{~m}$, known

malignancy, chronic liver or kidney disease major upper GI surgery,

psychiatric disease including

substance abuse, ED, mental

retardation or other intellectual

disability.

Aspiration Therapy: $\mathrm{n}=25$, follow-up $\mathrm{n}=20$; $23 \mathrm{~F}(92 \%), \mu 48 \mathrm{y}$, (range: 33$65 \mathrm{y})$, BMI $\mu 39.8+4.3 \mathrm{~kg} / \mathrm{m} 2$.

Ponce et al., 2012 (49)

RCT-;2010- 20

Exclusion: peptic ulcer, erosive esophagitis, hiatus hernia $>2 \mathrm{~cm}$,

etc.

USA

IGB: $n=21$, follow-up $n=20,17 F$

$(81 \%), \mu 38.9+9.1 \mathrm{y}, \mathrm{BMI}$

$\mu 34.7+2.6 \mathrm{~kg} / \mathrm{m} 2$.

\section{Reimao, 2018 (48)}

Prospective observational study-;

2014-2016.

Exclusion: any contradictions to IGB or impossibility of follow-up. Brazil

IGB: $\mathrm{n}=36$ analysed (40 included); follow-up n=38, 28F (77.7\%), $\mu 45.3+7.6 y$ (range: $25-57 y$ ), BMI $\mu 32.9+2.0 \mathrm{~kg} / \mathrm{m} 2$.
Aspire Assist System.

Duration of Tx: 12m (participants had option to continue therapy for an additional $12 \mathrm{~m}$ )

Method: custom gastrostomy tube (Atube, Aspire Bariatrics) percutaneously inserted during gastroscopy under sedation. Drainage \& irrigation of the stomach performed 3x/day (76\% patients aspirated $3 \mathrm{x} /$ day), $20 \mathrm{mins}$ postmeal for $1-2 y$. Diet + exercise

counselling during $\mathrm{Tx}$.

Follow-up: 4 in $3 \mathrm{~m}$, then every $3 \mathrm{~m}$.

Cognitive behavioural therapy, 8

sessions.

ReShape Duo IGB System.

\section{Duration of Tx: $6 \mathrm{~m}$}

Method: $900 \mathrm{~mL}$ saline.

Follow-up: monthly-6m, bi-weekly-

48weeks.

Diet and exercise counselling.

\section{Orberra IGB.}

Duration of Tx: $6 \mathrm{~m}$

Method: inserted under general

anaesthesia, $600 \mathrm{~mL}$ saline and

methylene blue dye.

Follow-up: monthly (nutritionist)

Hypocaloric diet (1000kcal/day), 120

$\mathrm{min} /$ week physical activity suggested.

Caloric intake estimated by five $24-\mathrm{h}$

dietary recall on non-consecutive days for $1 \mathrm{~m}$.
Quality of life (12m follow-up):

EQ-5D baseline: $\mu 0.7+0.3$, follow-up: $\mu 0.9+0.1(\mathrm{p}<0.01)$, VAS

baseline: $\mu 63+15$, follow-up: $\mu 83+14,(p<0.01)$.

Weight loss $(\mathrm{kg})$ :

Baseline: $\mu 107.4+18.7 \mathrm{~kg}$, follow-up: $\mu 88.4+16.9 \mathrm{~kg}$, calculated

change: $-\mu 19 \mathrm{~kg}(\mathrm{p}<0.01)$

EWL\%: $\mu 44.5+28.8 \%$

Adverse events:

moderate pain: $n=13 / 25$, severe pain: $n=3 / 25$, hospital admission (suspected leakage): $n=2 / 25$, intra-abdominal leakage at

gastrostomy site: $n=1 / 25$, stoma site related problems: $n=3 / 25$.

Comorbidities: $(n=20)$

Baseline: T2DM: $n=7 / 20$, HTN: $n=8 / 20$, high cholesterol $n=2 / 20$,

mood disorder $n=6 / 20$, GERD $n=2 / 20$

Follow-up: T2DM $n=5 / 20$, HTN $n=7 / 20$, high cholesterol

$n=2 / 20$, mood disorder $n=6 / 20$, GERD $n=3 / 20$.

HbA1c (mmol $/ \mathrm{mol})$ Baseline; $\mu 47$ median (IQR 43-66), followup; 42, (36-64), $(\mathrm{p}<0.03)$,

Quality of Life (6m follow-up):

SF-36 domain scores: baseline physical functioning (83.6),

general health (73.9), MH (87.3). Follow-up: physical functioning (96.9), general health (80.7), MH (86.1). Calculated change:

physical: +13.3 , general health $+6.8, \mathrm{MH}-1.2$.

Weight loss:

Baseline: $\mu 100.8+11.6 \mathrm{~kg}$

EWL\%: $31.8 \%$.

Adverse events:

Hypoxia: $n=1 / 21$, nausea: $n=4 / 21$

Quality of life (6m follow-up):

SF-36 (validated Portuguese version) Physical Aspects (\%)

baseline: $\mu 70$, follow-up: 92 , calculated change: +22 . General

Health (\%) baseline: $\mu 43$, follow-up: $\mu 68$, calculated change: +25 .

MH (\%) baseline: $\mu 62$, follow-up: $\mu 79$, calculated change: +17 .

Data reported graphically.

Weight loss:

Baseline; $\mu 89.8+12.1 \mathrm{~kg}$, Follow-up: $\mu 77.5+14.6 \mathrm{~kg}$, calculated

change: $-\mu 12.3 \mathrm{~kg},(\mathrm{p}<0.001)$

TBWL\%: 13.7\%.

Adverse events:
Funding support by Scientific

Committee of Blekinge County Council.

nitial exploratory safety study

Funding by and written with

ssistance

from ReShape Medical Inc.

Contacted author for numerical

data, unable to provide.

numerical values of bar graph QOL). 


\begin{tabular}{|c|c|c|c|}
\hline & & colonisation of IGB: 2/40. & \\
\hline $\begin{array}{l}\text { Raftopoulos et al., 2017. (47) } \\
\text { Prospective observational, } \\
\text { nonrandomised study. } \\
\text { Exclusion: HF, COPD, previous } \\
\text { bariatric therapy, pregnancy, etc. } \\
\text { Greece. } \\
\text { IGB: } \mathrm{n}=12,58 \% \mathrm{~F}, \mu 41 \mathrm{y} \text { (range: } 18- \\
59 \mathrm{y}), \mathrm{BMI} \mu 36.1+3.2 \mathrm{~kg} / \mathrm{m} 2 \text {. }\end{array}$ & $\begin{array}{l}\text { Elipse Balloon. } \\
\text { Duration of Tx: } 4 \mathrm{~m} \text {. } \\
\text { Method: insertion via swallow with } \\
\text { water, } 550 \mathrm{~mL} \text { water containing citric } \\
\text { acid/potassium sorbate preservative. } \\
\text { Follow-up: fortnightly. } \\
\text { Diet and exercise program. }\end{array}$ & $\begin{array}{l}\text { Quality of Life (12m follow-up): } \\
\text { IWQOL-Lite score: Baseline } 65 \text {. Follow-up: } 58 \text {. Calculated } \\
\text { change: }-7 \text {. } \\
\text { Weight loss: } \\
\text { Baseline: } \mu 103.5+15.8 \mathrm{~kg} \text {. Calculated change: }-6.5 \mathrm{~kg} \text {. } \\
\text { EWL } \%: 17.6 \% \text {. } \\
\text { Adverse events: } \\
\text { Nausea: } \mathrm{n}=4 / 12 \text {, vomiting: } \mathrm{n}=1 / 12 \text {, abdominal cramping: } \mathrm{n}=1 / 12 \text {, } \\
\text { GERD: } \mathrm{n}=2 / 12 \text {, constipation: } \mathrm{n}=2 / 12 \text {. }\end{array}$ & $\begin{array}{l}\text { Raftopoulos received } \\
\text { consulting fees } \\
\text { for Allurion Technologies. }\end{array}$ \\
\hline $\begin{array}{l}\text { Guedes et al 2017(2), Guedes et al } \\
\text { 2016 (46) } \\
\text { Prospective observational study-; } \\
2011-2012 \text {. } \\
\text { Exclusion: T1/T2DM, pregnancy, } \\
\text { previous gastric surgery, hiatal hernia } \\
\text { p5cm, clotting disorders, potentially } \\
\text { bleeding gastrointestinal lesions, } \\
\text { alcoholism or use of drugs, previous } \\
\text { hx of psychiatric disorders, current } \\
\text { use of anti-depressants or other } \\
\text { psychiatric drug, and weight loss } \\
\text { treatment within the previous } 6 \mathrm{~m} . \\
\text { Brazil } \\
\text { IGB: } \mathrm{n}=50, \text { follow-up n=39, } 40 \mathrm{~F} \\
(80 \%), \mu 34.6+7.1 \mathrm{y}, \text { BMI } \mu 40+6.3 \\
\mathrm{~kg} / \mathrm{m} 2 .\end{array}$ & $\begin{array}{l}\text { Silmed Silicone IGB. } \\
\text { Duration of Tx: } 6 \mathrm{~m} \\
\text { Method: inserted under sedation, } \\
650 \mathrm{~mL} \text { saline solution }(0.9 \%) \text { and } \\
\text { 20mL methylene blue solution. } \\
\text { Follow-up: weeks } 0,8,16 \& 24 \text {. }\end{array}$ & $\begin{array}{l}\text { Quality of life (6m follow-up): } \\
\text { WHOQOL-BREF Physical domain baseline; } \mu 54.3+17.9(14.2- \\
92.8), \text { follow-up; } \mu 67.0+16.2,(25.0-100.0), \mathrm{p}<0.01, \\
\text { Psychological domain baseline; } \mu 55.9+17.2(12.5-91.6), \text { follow- } \\
\text { up; } \mu 64.5+19.9(16.6-95.8), \mathrm{p} 0.03 \text {. } \\
\text { Calculated change: physical: }+12.7 \text {, psychological: }+8.6 \text {. } \\
\text { Weight loss: } \\
\text { Calculated change: } \mu 11.7+9.6,(\mathrm{p}<0.0001) \\
\text { BMI: } \mu-4.4+3.5 \mathrm{~kg} / \mathrm{m} 2(\mathrm{p}<0.0001) \text {. } \\
\text { Adverse events: } \\
\text { Gastric intolerance: } \mathrm{n}=4 / 50, \text { balloon rupture: } \mathrm{n}=5 / 50, \text { uterus } \\
\text { cancer: } \mathrm{n}=1 / 50 . \\
\text { Depression/Anxiety }(6 \mathrm{~m} \text { follow-up): } \\
* \text { BDI: Baseline: } \mu 16(\mathrm{median}),(\mathrm{range}: 1-32), \text { follow-up; } \mu 6, \\
\text { (range: } 0-45), \text { change: } \mu 4.57 \pm 10.6,(\mathrm{p}=0.0019) . \\
\text { HADS-D baseline: } \mu 7 \text { (range: } 1-14), \text { follow-up: } 4(0-18), \text { change: } \\
1.82+5.16,(\mathrm{p}=0.0345) \text {. }\end{array}$ & $\begin{array}{l}\text { Funding by Silmed Silicone } \\
\text { Instrumental } \\
\text { Medico Ciurgico Hospital Ltda, } \\
\text { Rio de Janeiro, RJ, Brazil. The } \\
\text { funding body had no role in } \\
\text { study design, collection, } \\
\text { analysis, interpretation of data } \\
\text { or writing the manuscript. }\end{array}$ \\
\hline $\begin{array}{l}\text { Tayyem, Atkinson \& Martin, } \\
2014(45) \\
\text { Single centre, prospective study-; } \\
2010-2010 \text {. } \\
\text { Exclusion: no written consent, ESL. } \\
\text { Scotland. } \\
\text { IGB: } n=12,62 \% \mathrm{~F}, \mu 40 \mathrm{y}, \mathrm{BMI} \\
\mu 55.9 \mathrm{~kg} / \mathrm{m} 2 \text {. }\end{array}$ & $\begin{array}{l}\text { BioEnterics IGB (BIB) System } \\
\text { Duration of Tx: } 6 \mathrm{~m} \\
\text { Method: inserted under sedation, } \\
\text { 600mL saline containing methylene } \\
\text { blue. }\end{array}$ & $\begin{array}{l}\text { Quality of Life: } \\
\text { SF-36 domain scores: Baseline physical functioning: } 36.5 \text {, general } \\
\text { health: } 29 \text {. Follow-up: physical functioning: } 57.5 \text {, general health: } \\
63 \text {. Calculated change: physical functioning: }+21 \text {, general health: } \\
+34 \text {. } \\
\text { Weight loss: } \\
\text { Baseline: } \mu 156+21 \mathrm{~kg} \text {. Calculated change: }-15+12 \mathrm{~kg} \text {. } \\
\text { EWL\%: } 25.4 \% \text {. } \\
\text { Comorbidities: }\end{array}$ & $\begin{array}{l}\text { Authors contacted about IGB } \\
\text { data; data provided. }\end{array}$ \\
\hline
\end{tabular}


Baseline depression: $\mathrm{n}=10 / 12$.

\begin{tabular}{|l|l|l}
\hline Tayyem, Obondo Ali, 2011 (44) & BioEnterics IGB (BIB) System & Quality of Life (9m follow-up):
\end{tabular}

Tayyem, Obondo Ali, $2011(44)$
Prospective longitudinal study-; 2008-2010.

Exclusion: previous bariatric surgery/abdominal surgery, hiatus hernia, peptic ulcers, unfit for surgery/anaesthesia.

Scotland.

IGB: $\mathrm{n}=17,65 \% \mathrm{~F}, \mu 40.9 \mathrm{y}, \mathrm{BMI}$ $\mu 61.4+8.3 \mathrm{~kg} / \mathrm{m} 2$.

\section{BioEnterics IGB (BIB) System}

Duration of Tx: $6 \mathrm{~m}$

Method: inserted under sedation,

$600 \mathrm{~mL}$ saline containing methylene

blue.

Follow-up: quarterly.
SF-36 domain scores: Baseline physical functioning: 35, general health: 28. Follow-up: physical functioning: 72, general health:

70. Calculated change: physical functioning: $+37, p<0.041$

general health: $+42, \mathrm{p}<0.021$

Data reported graphically.

Weight loss:

Baseline: $\mu 172+19.5 \mathrm{~kg}$. Calculated change: $-\mu 25.6+14.4 \mathrm{~kg}$, $\mathrm{p}<0.001$.

EWL\%: $26.2+14 \%$.

Adverse events:

Nausea: $n=4 / 17$, vomiting: $n=4 / 17$.

Comorbidities:

Baseline: DM: $n=3 / 17$, HTN: $n=6 / 17$, hyperlipidaemia: $n=3 / 17$, IHD: $n=4 / 17$, OSA: $n=2 / 17$.

Follow-up:

DM 1/17, HTN 1/17, hyperlipidaemia 1/17, IHD 2/17, OSA 1/17.

Quality of life (12m follow-up):

Thompson et al, 2017 (57)

RCT-; 2012-2015.

Exclusion: hx of gastrointestinal disease or previous abdominal

surgery increasing the risk of A-tube placement, previous bariatric surgery chronic abdominal pain, serious

CVD, medication significantly

Aspire Assist System

IWQOL Total Score change: $\mu 6.2+13.4$, Physical Function score

Duration of Tx: $52 \mathrm{w}$

Method: Endoscopically placed

percutaneous gastrostomy tube $(15 \mathrm{~cm}$

change: $7.1+15.5$.

Weight loss:

Baseline: $116.9 \pm 21.2$, Change: $\mu 14.2+11.3 \mathrm{~kg}$

EBWL\%: $\mu 37.2+27.5 \%$

external device to facilitate drainage of
$30 \%$ of calories consumed 20mins post- Adverse Events:

meal.

impacting on weight loss or weight Follow-up: week 0,

gain and hx of major depressive,

psychiatric or eating disorders.

USA

$2,6,10,14,20,24,28,32,36,40,44,48$ \&

52.

Diet and lifestyle counselling

program.

Aspiration therapy: $\mathrm{n}=82(\mathrm{n}=26$

Abdominal pain within 4 weeks: $n=42 / 111$, peristomal granulation

tissue: $n=45 / 111$, peristomal irritation: $n=19 / 111$,

nausea/vomiting: $n=19 / 111$, intermittent abdominal discomfort:

$n=18 / 111$, peristomal bacterial infection: $n=15 / 111$, dyspepsia:

$n=7 / 111$, peristomal inflammation: $n=6 / 111$.

Serious adverse events: $n=4 / 111$, severe abdominal pain: $n=1 / 111$,
Author contacted for numerical

alues of graphs.

Orlistat $120 \mathrm{mg}$ prescribed $3 / \mathrm{d}$

for weight loss, access to

helpline and referrals to

gym/slimming activities

provided pre-procedure.

withdrew pre-enrolment, $\mathrm{n}=29$

dropped out), $68 \mathrm{~F}(82.9 \%)$

$\mu 43.5+10.2 \mathrm{y}, \mathrm{BMI} \mu 42.4+5.0$

$\mathrm{kg} / \mathrm{m} 2$.

peritonitis: $n=1 / 111$, pre-pyloric ulcer: $n=1 / 111$, a-tube

replacement (skin port malfunction): $n=1 / 111$.

Comorbidities:

HbA1c baseline; $\mu 5.7+0.5$, change: $0.36 \%(\mathrm{p}<0.0001), \mathrm{TG}$

baseline: $\mu 140.8+81.7$, change: $9.9 \%(\mathrm{p}=0.02)$,

SBP baseline: $\mu 12.2+13.3$, change: $1.2 \%(\mathrm{p}=0.38)$

DBP baseline; $\mu 78.8+8.9$, change: $2.6 \%(\mathrm{p}=0.06)$,

LDL; baseline $\mu 115.4+32.8$, change: $4.2 \%(p=0.06 \%)$

Funded by Aspire Bariatrics -

performed statistical analysis \&

assisted preparing the

manuscript.

Participants were permitted to

continue in the study for an

dditional $48 \mathrm{~m}$ if they lost and

maintained at least $10 \%$ of their

body weight from baseline. 
Footnote:

BDI: Beck Depression Inventory; BDI-II: Beck Depression Inventory II; BFM: Body fat mass; BMI: body mass index; BW: Body weight; CRP: C-reactive Protein; DBP: Diastolic blood pressure; DLD: dyslipidaemia; DM: diabetes mellitus; ED: Eating disorder; EQ-5D: European Quality of life measurement questionnaire; ESL: English as a second language; EW: Excess weight; EWL: excess weight loss; FBGL: Fasting blood glucose; GIQLI: Gastrointestinal Quality of Life Index; Hx: history; HDAS-A: Hospital Anxiety and Depression Scale (Anxiety score); HDAS-D: Hospital Anxiety and Depression Scale (Depression Score); HTN: hypertension; IBD: inflammatory bowel disease; IGB: intragastric balloon; IQR: interquartile range; IWQOL-Lite: Impact of Weight on QOL-Lite; LDL: low density lipoprotein; MH: mental health; MI: myocardial infarction; MS: Metabolic Syndrome; NR: not reported; QOL: quality of life; SBP: Systolic blood pressure; SD: standard deviation; SF-12: Quality Metric's Short Form; SF-36: 36-Item Short-Form Health Survey; TBWL: Total body weight loss; TC: Total Cholesterol; TG: Triglycerides; TOGA: transoral gastroplasty; VAS: Visual Analogue Scale; WC: Waist circumference; WL: Weight loss.

QOL/Mental health assessment tools (indication of improvement):

BDI: score decreases [58]

BDI-II: score decreases [59]

EQ-5D: score increases[60] GIQLI: score increases. 4 is the most desirable option, 0 is the least desirable option [61]

HDAS: score decreases[62]

IWQOL-BREF- A higher score indicates an improved quality of life [63]

IWQOL-Lite: score increases. Scores range from 0 to 100, with 100 representing the best quality of life [64]

IWQOL- higher scores indicated lower levels of functioning and QOL [65]

SF-12: score decreases [66]

SF-36: A higher score indicates a better health status [67] 
This is a post-peer-review, pre-copyedit version of an article published in Obesity Surgery. 
Table S3: Risk of bias assessments and justifications using Academy of Nutrition and Dietetics Quality Criteria Checklist.

\begin{tabular}{|c|c|c|c|c|c|c|c|c|c|c|c|}
\hline Study ID & $\begin{array}{l}\text { 1. Was the } \\
\text { research } \\
\text { question } \\
\text { clearly stated? }\end{array}$ & $\begin{array}{l}\text { 2. Was the } \\
\text { selection of } \\
\text { study } \\
\text { subjects } \\
\text { free from } \\
\text { bias? }\end{array}$ & $\begin{array}{l}\text { 3. Were } \\
\text { study } \\
\text { groups } \\
\text { comparable } \\
?\end{array}$ & $\begin{array}{l}\text { 4. Was } \\
\text { method } \\
\text { of } \\
\text { handling } \\
\text { withdraw } \\
\text { als } \\
\text { described } \\
\text { ? }\end{array}$ & $\begin{array}{l}\text { 5. Was } \\
\text { blinding } \\
\text { used to } \\
\text { prevent } \\
\text { introducti } \\
\text { on of } \\
\text { bias? }\end{array}$ & $\begin{array}{l}\text { 6. Were the } \\
\text { intervention/thera } \\
\text { peutic } \\
\text { regimens/exposur } \\
\text { e factor or } \\
\text { procedure and any } \\
\text { comparisons } \\
\text { described in } \\
\text { detail? Were } \\
\text { intervening factors } \\
\text { described? }\end{array}$ & $\begin{array}{l}\text { 7. Were } \\
\text { outcomes } \\
\text { clearly } \\
\text { defined } \\
\text { and the } \\
\text { measureme } \\
\text { nts valid } \\
\text { and } \\
\text { reliable? }\end{array}$ & $\begin{array}{l}\text { 8. Was the } \\
\text { statistical } \\
\text { analysis } \\
\text { appropriat } \\
\text { e for the } \\
\text { study } \\
\text { design and } \\
\text { type of } \\
\text { outcome } \\
\text { indicators? }\end{array}$ & $\begin{array}{l}\text { 9. Are } \\
\text { conclusion } \\
\text { s supported } \\
\text { by results } \\
\text { with biases } \\
\text { and } \\
\text { limitations } \\
\text { taken into } \\
\text { considerati } \\
\text { on? }\end{array}$ & $\begin{array}{l}\text { 10. Is bias } \\
\text { due to study's } \\
\text { funding or } \\
\text { sponsorship } \\
\text { unlikely? }\end{array}$ & $\begin{array}{l}\text { Overall study } \\
\text { quality } \\
\text { (positive/negative/n } \\
\text { eutral) }\end{array}$ \\
\hline \multirow{2}{*}{$\begin{array}{l}\text { Ahmed } \\
\text { et al., } \\
2019\end{array}$} & Yes & No & $\mathrm{N} / \mathrm{A}$ & No & N/A & Unclear & Unclear & No & Unclear & Unclear & Neutral \\
\hline & $\begin{array}{l}\text { "To evaluate } \\
\text { the effect of } \\
\text { weight loss } \\
\text { and aspects of } \\
\text { quality of life } \\
\text { after BIB } \\
\text { insertion." } \\
\text { p.42. 1.3 } \\
\text { Participants } \\
\text { not specified. }\end{array}$ & $\begin{array}{l}2.3 \text { No } \\
\text { comorbidit } \\
\text { y data only } \\
\text { age, weight } \\
\& \text { gender. } \\
2.4 \\
\text { Included } \\
\text { only single } \\
\text { females } \\
20-40 y . \\
\text { Selection } \\
\text { method not } \\
\text { stated. }\end{array}$ & N/A & $\begin{array}{l}4.2 \\
\text { Withdraw } \\
\text { al/ lost to } \\
\text { follow-up } \\
\text { not } \\
\text { reported. } \\
4.3 \\
\text { Enrolled } \\
\text { subjects } \\
\text { not } \\
\text { accounte } \\
\text { d for. }\end{array}$ & N/A & $\begin{array}{l}6.4 \text { No drop out or } \\
\text { adverse events } \\
\text { reported. } \\
\text { Compliance } \\
\text { unclear. } 6.5 \\
\text { Dietary control } \\
\text { not described for } \\
\text { IGB group. }\end{array}$ & $\begin{array}{l}\text { 7.1 QoL } \\
\text { tool type } \\
\text { not } \\
\text { reported. } \\
7.4 \\
\text { Aspects of } \\
\text { domains } \\
\text { reported as } \\
\text { outcomes } \\
\text { rather than } \\
\text { outcomes } \\
\text { of } \\
\text { domains. }\end{array}$ & $\begin{array}{l}8.1 \\
\text { Changes } \\
\text { reported in } \\
\text { categorical } \\
\text { variables. } \\
8.2 \text { No } \\
\text { discussion } \\
\text { of non- } \\
\text { parametric } \\
\text { results. } \\
\text { Only } \\
\text { means, no } \\
\text { SD. } 8.4 \\
\text { Nil } \\
\text { intention } \\
\text { to treat. } \\
8.5 \text { No } \\
\text { multivariat } \\
\text { e analysis } \\
\text { or analysis } \\
\text { for } \\
\text { confounde } \\
\text { rs. }\end{array}$ & $\begin{array}{l}9.1 \\
\text { Discussed } \\
\text { findings. } \\
9.2 \\
\text { Limitation } \\
\text { s briefly } \\
\text { discussed; } \\
\text { no bias } \\
\text { discussed. }\end{array}$ & $\begin{array}{l}10.1 \text { "No any } \\
\text { sources of } \\
\text { funding for } \\
\text { the research." } \\
10.2 \text { No } \\
\text { conflicts of } \\
\text { interest to } \\
\text { declare. } \\
\text { Author is } \\
\text { "Manager of } \\
\text { Hospital for } \\
\text { Endoscopic } \\
\text { and Bariatric } \\
\text { Surgery" } \\
\text { p.42. }\end{array}$ & \\
\hline \multirow{2}{*}{$\begin{array}{l}\text { De } \\
\text { Castro et } \\
\text { al., } 2010\end{array}$} & Yes & Yes & Yes & Yes & Yes & Yes & Yes & Yes & Unclear & Unclear & Positive \\
\hline & $\begin{array}{l}\text { "To evaluate } \\
\text { the efficacy, }\end{array}$ & $\begin{array}{l}2.4 " 40 \\
\text { subjects } \\
\text { referred to }\end{array}$ & $\begin{array}{l}\text { 3.3 Unclear- } \\
\text { not stated. } \\
3.4 \text { No }\end{array}$ & $\begin{array}{l}4.4 \\
\text { Unclear. }\end{array}$ & & & $\begin{array}{l}7.4 \text { Not all } \\
\text { measureme }\end{array}$ & $\begin{array}{l}8.2 \text { Non- } \\
\text { parametric } \\
\text { test }\end{array}$ & & $\begin{array}{l}10.2 \mathrm{No} \\
\text { conflict } \\
\text { declaration }\end{array}$ & \\
\hline
\end{tabular}




\begin{tabular}{|c|c|c|c|c|c|c|c|c|c|c|c|}
\hline & $\begin{array}{l}\text { safety, and } \\
\text { tolerance } \\
\text { of this new } \\
\text { device } \\
\text { compared to } \\
\text { the saline- } \\
\text { filled BIB }{ }^{\circledR} \\
\text { balloon" } \\
1.3 \text { Population } \\
\text { not specified. }\end{array}$ & $\begin{array}{l}\text { place a } \\
\text { gastric } \\
\text { balloon" - } \\
\text { sampling } \\
\text { unclear. }\end{array}$ & $\begin{array}{l}\text { adjustments } \\
\text { in statistical } \\
\text { analysis. }\end{array}$ & & & & $\begin{array}{l}\text { nt methods } \\
\text { described. }\end{array}$ & $\begin{array}{l}\text { discussed, } \\
\text { mean, SD } \\
\text { reported. } \\
8.3 \text { p- } \\
\text { values } \\
\text { reported } \\
\text { only in } \\
\text { text. } \\
8.5 \text { No } \\
\text { adjustment } \\
\text { for } \\
\text { confounde } \\
\text { rs. }\end{array}$ & & $\begin{array}{l}\text { present. } 10.1 \\
\text { FISS grant. }\end{array}$ & \\
\hline \multirow{2}{*}{$\begin{array}{l}\text { Familiari } \\
\text { et al., } \\
2011\end{array}$} & Yes & Yes & Unclear & Yes & N/A & Yes & Yes & No & Yes & No & Positive \\
\hline & $\begin{array}{l}\text { "To evaluate } \\
\text { the safety and } \\
\text { efficacy of } \\
\text { TOGA at } 12- \\
\text { month follow- } \\
\text { up." } 1.3 \\
\text { Population not } \\
\text { specified. }\end{array}$ & $\begin{array}{l}2.4 \text { Offered } \\
\text { the } \\
\text { treatment } \\
\text { at bariatric } \\
\text { clinic } \\
\text { 'consecutiv } \\
\text { e } \\
\text { sampling". }\end{array}$ & $\begin{array}{l}\text { 3.4 Unclear- } \\
\text { no } \\
\text { comparison } \\
\text { between } \\
\text { groups. } \\
\text { Differed in } \\
\text { health } \\
\text { status. } \\
\text { Statistical } \\
\text { analysis } \\
\text { adjustments } \\
\text { not stated. }\end{array}$ & $\begin{array}{l}4.221 \% \\
\text { of } \\
\text { patients } \\
\text { lost to } \\
\text { follow- } \\
\text { up. } \\
4.4 \\
\text { Unlcear. }\end{array}$ & $\begin{array}{l}5.3 \\
\text { Unclear if } \\
\text { measurem } \\
\text { ent of } \\
\text { outcomes } \\
\text { \& risk } \\
\text { factors } \\
\text { blinded. }\end{array}$ & & $\begin{array}{l}7.2 \\
\text { IWQOL } \\
\text { and SF-36 } \\
\text { used for } \\
\text { QoL, } \\
\text { scores not } \\
\text { reported. } \\
7.4 \text { Not all } \\
\text { data } \\
\text { collection } \\
\text { methods/ } \\
\text { measures } \\
\text { described. }\end{array}$ & $\begin{array}{l}8.1 \\
\text { Inadequate } \\
\text { description } \\
\text { - statistical } \\
\text { program \& } \\
\text { level of } \\
\text { significanc } \\
\text { e not } \\
\text { reported. } \\
8.2 \\
\text { Statistical } \\
\text { tests not } \\
\text { described- } \\
\text { mean }+ \\
\text { SD } \\
\text { reported- } \\
\text { no } \\
\text { discussion } \\
\text { of non- } \\
\text { parametric } \\
\text { variables. } \\
8.3 \text { p- } \\
\text { values } \\
\text { reported } \\
\text { but level } \\
\text { of } \\
\text { significanc } \\
\text { e not }\end{array}$ & & $\begin{array}{l}10.1 \\
\text { Funded by Sa } \\
\text { tiety Inc. } 10.2 \\
\text { Sponsor } \\
\text { collaborated } \\
\text { with } \\
\text { investigators } \\
\text { in data } \\
\text { collection \& } \\
\text { analysis. }\end{array}$ & \\
\hline
\end{tabular}




\begin{tabular}{|c|c|c|c|c|c|c|c|c|c|c|c|}
\hline & & & & & & & & $\begin{array}{l}\text { discussed. } \\
8.4 \mathrm{Nil} \\
\text { intent to } \\
\text { treat. } \\
8.5 \mathrm{No} \\
\text { adjustment } \\
\text { for } \\
\text { confounde } \\
\text { rs. } \\
8.6 \\
\text { Clinical } \\
\text { significanc } \\
\text { e not } \\
\text { reported. } \\
\end{array}$ & & & \\
\hline \multirow{2}{*}{$\begin{array}{l}\text { Fuller et } \\
\text { al., } 2013\end{array}$} & Yes & Yes & N/A & Unclear & N/A & Yes & Yes & Unclear & Yes & No & Positive \\
\hline & $\begin{array}{l}\text { "Evaluated } \\
\text { the efficacy } \\
\text { and safety of } \\
\text { an IGB in } \\
\text { obese } \\
\text { individuals } \\
\text { with } \\
\text { metabolic } \\
\text { syndrome } \\
\text { (MS)". - } \\
\text { p1562. } \\
\text { Population, } \\
\text { intervention, } \\
\text { outcomes } \\
\text { stated (1.1- } \\
\text { 1.3). }\end{array}$ & $\begin{array}{l}2.4 \\
\text { Selection } \\
\text { method not } \\
\text { stated. }\end{array}$ & N/A & $\begin{array}{l}4.3 \\
\text { Tables do } \\
\text { not } \\
\text { describe } \\
\text { number } \\
\text { of } \\
\text { participan } \\
\text { ts } \\
\text { analysed. }\end{array}$ & & & $\begin{array}{l}7.6 \text { No } \\
\text { confoundin } \\
\text { g variables } \\
\text { considered. }\end{array}$ & $\begin{array}{l}8.1 \text { Results } \\
\text { expressed } \\
\text { only as } \\
\text { mean and } \\
\text { CI, no } \\
\text { discussion } \\
\text { about non- } \\
\text { parametric } \\
\text { values. } 8.4 \\
\text { Unclear. } \\
8.5 \text { No } \\
\text { multivariat } \\
\text { e analysis. } \\
8.6 \\
\text { clinically } \\
\text { significanc } \\
\text { e is } \\
\text { referred to } \\
\text { with the } \\
\text { QoL \& } \\
\text { weight } \\
\text { changes. }\end{array}$ & & $\begin{array}{l}\text { 10.1 Funding } \\
\text { received. } 10.2 \\
\text { Conflicts of } \\
\text { interests: } 1 \\
\text { author } \\
\text { employee of } \\
\text { Allergan } \\
\text { institute. }\end{array}$ & \\
\hline \multirow{2}{*}{$\begin{array}{l}\text { Alfredo } \\
\text { et al., } \\
2014\end{array}$} & Yes & Yes & N/A & Yes & N/A & No & Yes & No & Unclear & No & Neutral \\
\hline & $\begin{array}{l}\text { "To } \\
\text { investigate the } \\
\text { efficacy of }\end{array}$ & $\begin{array}{l}2.4 \\
\text { Convenien } \\
\text { ce }\end{array}$ & $\begin{array}{l}\text { One study } \\
\text { group. }\end{array}$ & $\begin{array}{l}4.2 \\
\text { Dropouts } \\
\text { described }\end{array}$ & & $\begin{array}{l}6.4 \text { No } \\
\text { comparison of } \\
\text { patients that }\end{array}$ & & $\begin{array}{l}8.1 \text { Data } \\
\text { tables do } \\
\text { not include }\end{array}$ & & $\begin{array}{l}\text { 10.1 Funding } \\
\text { not reported. } \\
10.2 \text { No }\end{array}$ & \\
\hline
\end{tabular}

This is a post-peer-review, pre-copyedit version of an article published in Obesity Surgery. 


\begin{tabular}{|c|c|c|c|c|c|c|c|c|c|c|c|}
\hline & $\begin{array}{l}\text { multiple } \\
\text { balloon } \\
\text { treatment in } \\
\text { the long term } \\
\text { ( } 6 \text { years) in } \\
\text { terms of } \\
\text { weight loss, } \\
\text { influence of } \\
\text { comorbidities } \\
\text { and QOL in } \\
\text { patients } \\
\text { refusing } \\
\text { surgery". - } \\
\text { p.307. } \\
\text { Population, } \\
\text { outcomes \& } \\
\text { intervention } \\
\text { stated (1.1- } \\
1.3) \text {. }\end{array}$ & $\begin{array}{l}\text { sampling } \\
\text { p.308 - } \\
\text { Recruited } \\
\text { from } \\
\text { prospective } \\
\text { database. }\end{array}$ & & $\begin{array}{l}\text {. Final } \\
\text { follow-up } \\
\text { analysis } \\
\text { on } 74 \% \text {. } \\
4.3 \\
\text { Tables \& } \\
\text { figures do } \\
\text { not state } \\
\text { number } \\
\text { of } \\
\text { participan } \\
\text { ts } \\
\text { included } \\
\text { in } \\
\text { analysis. }\end{array}$ & & $\begin{array}{l}\text { underwent }>2 \\
\text { IGBs to those that } \\
\text { had } 2 \text {. }\end{array}$ & & $\begin{array}{l}\text { participant } \\
\text { numbers. } \\
8.2 \\
\text { Reported } \\
\text { mean and } \\
\text { SE. } \\
8.4 \text { Nil } \\
\text { intention } \\
\text { to treat. } \\
8.5 \mathrm{No} \\
\text { adjustment } \\
\text { s for } \\
\text { confounde } \\
\text { rs. }\end{array}$ & & $\begin{array}{l}\text { conflicts of } \\
\text { interest } \\
\text { reported. }\end{array}$ & \\
\hline \multirow{2}{*}{$\begin{array}{l}\text { Guedes } \\
\text { et al., } \\
2019\end{array}$} & Yes & Yes & N/A & No & N/A & Unclear & Yes & Unclear & Yes & Unclear & Neutral \\
\hline & $\begin{array}{l}\text { "To evaluate } \\
\text { the changes in } \\
\text { body weight, } \\
\text { total and } \\
\text { central body } \\
\text { adiposity, } \\
\text { dietary intake, } \\
\text { habitual } \\
\text { physical } \\
\text { activity and } \\
\text { quality of life, } \\
\text { of patients } \\
\text { with obesity } \\
\text { submitted to } \\
\text { IGB treatment } \\
\text { for } 6 \text { months." } \\
\text { - p.843. } \\
\text { Outcomes, } \\
\text { participants \& } \\
\text { intervention } \\
\text { stated (1.1- } \\
\text { 1.3). }\end{array}$ & $\begin{array}{l}\text { "Potential } \\
\text { participants } \\
\text { were } \\
\text { recruited } \\
\text { among } \\
\text { patients } \\
\text { who had } \\
\text { already } \\
\text { scheduled } \\
\text { the } \\
\text { placement } \\
\text { of } \\
\text { nonadjusta } \\
\text { ble IGB" - } \\
\text { p.844 } \\
\text { convenient } \\
\text { sample. }\end{array}$ & $\begin{array}{l}\text { One group } \\
\text { in study. }\end{array}$ & $\begin{array}{l}4.2 \\
\text { Withdraw } \\
\text { al reasons } \\
\text { not } \\
\text { specified. }\end{array}$ & $\begin{array}{l}\text { No } \\
\text { control } \\
\text { group, } \\
\text { blinding } \\
\text { not } \\
\text { possible. }\end{array}$ & $\begin{array}{l}6.4 \text { Compliance } \\
\text { not reported. }\end{array}$ & & $\begin{array}{l}8.1 \\
\text { Shapiro } \\
\text { Wilk test } \\
\text { for } \\
\text { normality. } \\
8.2 \text { Mean } \\
\& \text { SEM for } \\
\text { parametric } \\
\text { data } \\
\text { (inaccurate } \\
\text { reporting } \\
\text { of data). } \\
8.3 \mathrm{P}= \\
<0.05 \text {. } \\
8.5 \\
\text { Confounde } \\
\text { rs } \\
\text { adjustment } \\
\text { s not stated } \\
\text {-p846. }\end{array}$ & & $\begin{array}{l}\text { 10.1 Funding } \\
\text { not stated. } \\
\text { 10.2 Declared } \\
\text { no conflicts. }\end{array}$ & \\
\hline
\end{tabular}

This is a post-peer-review, pre-copyedit version of an article published in Obesity Surgery. 


\begin{tabular}{|c|c|c|c|c|c|c|c|c|c|c|c|}
\hline \multirow{2}{*}{$\begin{array}{l}\text { Guedes } \\
\text { et al., } \\
2016\end{array}$} & Yes & Yes & N/A & Yes & N/A & Yes & Yes & No & Yes & Yes & Positive \\
\hline & $\begin{array}{l}\text { "Investigate } \\
\text { the effects of } \\
\text { a } 6 \text {-month } \\
\text { treatment with } \\
\text { IGB on body } \\
\text { composition } \\
\text { and } \\
\text { depressive/an } \\
\text { xiety } \\
\text { symptoms in } \\
\text { obese } \\
\text { individuals } \\
\text { with MS". } \\
1.3 \text { Setting not } \\
\text { stated. }\end{array}$ & $\begin{array}{l}2.4 \\
\text { "consecuti } \\
\text { ve sample } \\
\text { of } 50 \\
\text { patients } \\
\text { who sought } \\
\text { treatment } \\
\text { for obesity } \\
\text { and MS". }\end{array}$ & $\begin{array}{l}\text { One group } \\
\text { in study. }\end{array}$ & $\begin{array}{l}4.221 \% \\
\text { lost to } \\
\text { follow } \\
\text { up, all } \\
\text { withdraw } \\
\text { als } \\
\text { described } \\
\text {. }\end{array}$ & & $\begin{array}{l}6.5 \text { Ancillary } \\
\text { treatments not } \\
\text { discussed. }\end{array}$ & $\begin{array}{l}\text { 7.6 Other } \\
\text { factors not } \\
\text { accounted } \\
\text { for. }\end{array}$ & $\begin{array}{l}8.2 \text { Only } \\
\text { means } \\
\text { reported, } \\
\text { no } \\
\text { discussion } \\
\text { of non- } \\
\text { parametric } \\
\text { data. } \\
8.4 \text { Nil } \\
\text { intention } \\
\text { to treat. } \\
8.5 \text { No } \\
\text { multivariat } \\
\text { e } \\
\text { regression }\end{array}$ & $\begin{array}{l}9.1 \\
\text { Discussion } \\
\text { included. } \\
9.2 \\
\text { Limitation } \\
\text { s } \\
\text { discussed. }\end{array}$ & $\begin{array}{l}\text { 10.1 Funding } \\
\text { not stated. } \\
10.2 \text { No } \\
\text { conflicts } \\
\text { declaration } \\
\text { stated. }\end{array}$ & \\
\hline \multirow{2}{*}{$\begin{array}{l}\text { Guedes } \\
\text { et al., } \\
2017\end{array}$} & Yes & Yes & N/A & Yes & N/A & Yes & Yes & Yes & Yes & Unclear & Positive \\
\hline & $\begin{array}{l}\text { "To } \\
\text { investigate the } \\
\text { effect of } 6 \\
\text { months of } \\
\text { treatment with } \\
\text { an intragastric } \\
\text { balloon (IGB) } \\
\text { on health- } \\
\text { related quality } \\
\text { of life } \\
\text { (HRQOL) and } \\
\text { its relation to } \\
\text { changes in } \\
\text { body fat in } \\
\text { obese } \\
\text { individuals } \\
\text { with } \\
\text { metabolic } \\
\text { syndrome } \\
\text { (MS)." }\end{array}$ & $\begin{array}{l}2.4 \\
\text { Consecutiv } \\
\text { e sampling. }\end{array}$ & $\begin{array}{l}\text { One group } \\
\text { in study }\end{array}$ & $\begin{array}{l}4.222 \% \\
\text { patients } \\
\text { withdrew } \\
\text { from } \\
\text { study. }\end{array}$ & $\begin{array}{l}\text { One } \\
\text { group in } \\
\text { study. }\end{array}$ & $\begin{array}{l}6.5 \text { Co- } \\
\text { interventions not } \\
\text { reported. }\end{array}$ & $\begin{array}{l}\text { 7.6 Other } \\
\text { factors not } \\
\text { accounted } \\
\text { for. }\end{array}$ & $\begin{array}{l}8.4 \text { Nil } \\
\text { intention } \\
\text { to treat. }\end{array}$ & & $\begin{array}{l}\text { 10.1 Funding } \\
\text { "not } \\
\text { applicable". } \\
10.2 \text { No } \\
\text { conflicts of } \\
\text { interest } \\
\text { reported. }\end{array}$ & \\
\hline \multirow{2}{*}{$\begin{array}{l}\text { Machytk } \\
\text { a et al., } \\
2017\end{array}$} & Yes & Yes & N/A & Yes & N/A & Yes & Unclear & No & Yes & Yes & Neutral \\
\hline & $\begin{array}{l}\text { "To assess the } \\
\text { safety of } \\
\text { Elipse and to }\end{array}$ & $\begin{array}{l}2.3 \text { No } \\
\text { demograph } \\
\text { ics. }\end{array}$ & $\begin{array}{l}\text { One group } \\
\text { in study. }\end{array}$ & & $\begin{array}{l}\text { One } \\
\text { group in } \\
\text { study. }\end{array}$ & 6.3 4-months. & $\begin{array}{l}7.34- \\
\text { months - }\end{array}$ & $\begin{array}{l}8.1 \\
\text { Insufficien } \\
\mathrm{t}\end{array}$ & $\begin{array}{l}9.1 \\
\text { Discussed } \\
\text { findings. }\end{array}$ & $\begin{array}{l}\text { 10.1 Funding } \\
\text { not stated. } \\
10.2 \text { Two }\end{array}$ & \\
\hline
\end{tabular}




\begin{tabular}{|c|c|c|c|c|c|c|c|c|c|c|c|}
\hline & $\begin{array}{l}\text { measure its } \\
\text { effects on } \\
\text { weight loss, } \\
\text { metabolic } \\
\text { parameters } \\
\text { and quality of } \\
\text { life" } 1.3 \\
\text { Population not } \\
\text { stated. }\end{array}$ & $\begin{array}{l}2.4 \text { Sample } \\
\text { from } 2 \\
\text { hospitals - } \\
\text { consecutiv } \\
\text { e sample. } \\
\text { Unclear if } \\
\text { representati } \\
\text { ve sample. }\end{array}$ & & & & & $\begin{array}{l}\text { insufficient } \\
\text { 7.4 } \\
\text { Validated } \\
\text { measures - } \\
\text { accuracy } \\
\text { questioned. } \\
7.6 \text { Other } \\
\text { factors not } \\
\text { accounted. }\end{array}$ & $\begin{array}{l}\text { informatio } \\
\text { n. } \\
8.4 \text { Nil } \\
\text { intention } \\
\text { to treat. } \\
8.5 \text { No } \\
\text { adjustment } \\
\text { for } \\
\text { confounde } \\
\text { rs. }\end{array}$ & $\begin{array}{l}9.2 \\
\text { Limitation } \\
\mathrm{s} \\
\text { discussed. }\end{array}$ & $\begin{array}{l}\text { authors } \\
\text { received } \\
\text { consulting } \\
\text { fees, } 1 \text { is a } \\
\text { consultant } \\
\text { and } 2 \text { are } \\
\text { shareholders } \\
\text { in Allurion } \\
\text { Technologies. }\end{array}$ & \\
\hline \multirow{2}{*}{$\begin{array}{l}\text { Marinos } \\
\text { et al., } \\
2014\end{array}$} & Unclear & Yes & Unclear & Yes & N/A & Yes & Yes & No & Unclear & No & Neutral \\
\hline & $\begin{array}{l}\text { To evaluate } \\
\text { the safety and } \\
\text { efficacy of the } \\
\text { clinical } \\
\text { procedure and } \\
\text { device. - } \\
\text { p.929. } \\
\text { Intervention } \\
\text { \& population } \\
\text { not stated } \\
(1.1,1.3) .\end{array}$ & $\begin{array}{l}2.4 \\
\text { Sampling } \\
\text { method not } \\
\text { reported. }\end{array}$ & 3.2 Unclear. & $\begin{array}{l}4.210 \% \\
\text { of } \\
\text { patients } \\
\text { withdrew, } \\
\text { reasons } \\
\text { stated. }\end{array}$ & $\begin{array}{l}\text { No } \\
\text { control } \\
\text { group, } \\
\text { blinding } \\
\text { not } \\
\text { possible. }\end{array}$ & & $\begin{array}{l}7.33- \\
\text { month data } \\
\text { not } \\
\text { sufficient. } \\
7.6 \text { Other } \\
\text { factors not } \\
\text { accounted } \\
\text { for. }\end{array}$ & $\begin{array}{l}8.1 \\
\text { Inadequate } \\
\text { ly } \\
\text { described. } \\
8.2 \text { No } \\
\text { discussion } \\
\text { on non- } \\
\text { parametric } \\
\text { variables. } \\
8.4 \text { Nil } \\
\text { intention } \\
\text { to treat. } \\
8.5 \text { Nil } \\
\text { adjustment } \\
\text { s for } \\
\text { confounde } \\
\text { rs. }\end{array}$ & $\begin{array}{l}9.2 \\
\text { Limitation } \\
\text { s not } \\
\text { discussed. }\end{array}$ & $\begin{array}{l}10.1 \\
\text { Sponsored by } \\
\text { Baronova. } \\
10.22 \text { authors } \\
\text { were } \\
\text { consultants. }\end{array}$ & \\
\hline \multirow{2}{*}{$\begin{array}{l}\text { Moreno } \\
\text { et al., } \\
2008\end{array}$} & No & Yes & N/A & Yes & N/A & Yes & Yes & No & No & No & Neutra \\
\hline & $\begin{array}{l}\text { "6-month } \\
\text { results of } \\
\text { second phase } \\
\text { of the pilot } \\
\text { trial with the } \\
\text { TOGA } \\
\text { system" } \\
\text { Outcomes \& } \\
\text { population } \\
(1.2,1.3) .\end{array}$ & $\begin{array}{l}2.4 \text { Patients } \\
\text { recruited } \\
\text { into the } \\
\text { bariatric } \\
\text { practice - } \\
\text { sampling } \\
\text { unclear. }\end{array}$ & $\begin{array}{l}\text { Single arm } \\
\text { study }\end{array}$ & $\begin{array}{l}4.1 \\
\text { Follow- } \\
\text { up } \\
\text { described } \\
. \\
4.290 \% \\
\text { follow-up } \\
\text { rate, } \\
\text { withdraw } \\
\text { al stated. }\end{array}$ & & & $\begin{array}{l}7.6 \text { Other } \\
\text { factors not } \\
\text { accounted } \\
\text { for. }\end{array}$ & $\begin{array}{l}8.1 \\
\text { Insufficien } \\
\mathrm{t} \\
\text { informatio } \\
\mathrm{n} \& \\
\text { unclear } \\
\text { reporting } \\
\text { of QoL } \\
\text { Score. } 8.2 \\
\text { Results } \\
\text { reported as }\end{array}$ & $\begin{array}{l}9.2 \\
\text { Limitation } \\
\text { s not } \\
\text { discussed. }\end{array}$ & $\begin{array}{l}10.1 \text { Funded } \\
\text { by Satiety inc. } \\
10.2 \text { Conflicts } \\
\text { declared. }\end{array}$ & \\
\hline
\end{tabular}




\begin{tabular}{|c|c|c|c|c|c|c|c|c|c|c|c|}
\hline & & & & & & & & $\begin{array}{l}\text { mean }+ \\
\text { SEM. } \\
8.4 \text { Nil } \\
\text { intention } \\
\text { to treat. } \\
8.5 \text { No } \\
\text { adjustment } \\
\text { for } \\
\text { confounde } \\
\text { rs. } \\
8.7 \\
\text { Negative } \\
\text { findings in } \\
\text { IWQOL- } \\
\text { Lite } \\
\text { reported } \\
\text { but not } \\
\text { identified. }\end{array}$ & & & \\
\hline \multirow{2}{*}{$\begin{array}{l}\text { Mui et } \\
\text { al., } 2010\end{array}$} & Yes & Yes & N/A & Yes & N/A & Yes & Yes & Unclear & Unclear & unclear & Positive \\
\hline & $\begin{array}{l}\text { "To evaluate } \\
\text { the outcome } \\
\text { of IGB on } \\
\text { weight loss } \\
\text { and the } \\
\text { impact of it on } \\
\text { obesity- } \\
\text { related } \\
\text { illnesses and } \\
\text { quality of life } \\
\text { in obese } \\
\text { Chinese." - } \\
\text { p.1128. }\end{array}$ & $\begin{array}{l}2.4 \\
\text { Consecutiv } \\
\text { e sampling }\end{array}$ & $\begin{array}{l}\text { One group } \\
\text { in study. }\end{array}$ & 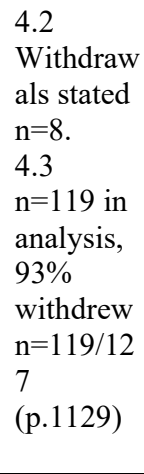 & & $\begin{array}{l}6.36 \& 12 \mathrm{~m} . \\
6.4 \text { lost to follow- } \\
\text { up/drop-out } \\
\text { excluded from } \\
\text { analysis. }\end{array}$ & $\begin{array}{l}7.6 \text { Other } \\
\text { factors } \\
\text { present but } \\
\text { not } \\
\text { accounted } \\
\text { for. }\end{array}$ & $\begin{array}{l}8.1 \\
\text { Student's t } \\
\text { test for } \\
\text { parametric } \\
\text { data \& } \\
\text { McNemar } \\
\text { test where } \\
\text { appropriat } \\
\text { e. } \\
8.2 \text { Only } \\
\text { mean \& } \\
\text { SD } \\
\text { reported. }\end{array}$ & $\begin{array}{l}9.2 \\
\text { Unclear, } \\
\text { limitations } \\
\text { on IGB not } \\
\text { the study. }\end{array}$ & $\begin{array}{l}\text { 10.1 Source } \\
\text { of funding not } \\
\text { reported. } 10.2 \\
\text { Conflicts of } \\
\text { interests not } \\
\text { discussed. }\end{array}$ & \\
\hline \multirow{2}{*}{$\begin{array}{l}\text { Norén, et } \\
\text { al., } 2016\end{array}$} & Yes & Yes & N/A & Yes & N/A & Yes & Yes & Yes & Yes & Yes & Positive \\
\hline & $\begin{array}{l}\text { "To evaluate } \\
\text { weight loss, } \\
\text { safety and } \\
\text { quality of life } \\
\text { with } \\
\text { AspireAssist } \\
\text { treatment for } \\
1 \text { to } 2 \text { years in }\end{array}$ & $\begin{array}{l}2.4 \\
\text { Consecutiv } \\
\text { e sample. }\end{array}$ & $\begin{array}{l}\text { One group } \\
\text { in study. }\end{array}$ & $\begin{array}{l}4.2 \\
\text { Withdraw } \\
\text { als } \\
\text { described } \\
\& \\
\text { number } \\
\text { stated, } \\
80 \%\end{array}$ & & $\begin{array}{l}\text { Number of } \\
\text { aspirations } \\
\text { measured but not } \\
\text { reported. }\end{array}$ & $\begin{array}{l}7.6 \text { Other } \\
\text { factors not } \\
\text { accounted } \\
\text { for }\end{array}$ & $\begin{array}{l}8.5 \text { No } \\
\text { adjustment } \\
\text { s for } \\
\text { confounde } \\
\text { rs. }\end{array}$ & & $\begin{array}{l}10.1 \text { Authors } \\
\text { received } \\
\text { research } \\
\text { support for } \\
\text { study from } \\
\text { the Scientific } \\
\text { committee of } \\
\text { Blekinge }\end{array}$ & \\
\hline
\end{tabular}




\begin{tabular}{|c|c|c|c|c|c|c|c|c|c|c|c|}
\hline & $\begin{array}{l}\text { obese } \\
\text { subjects". -p2. }\end{array}$ & & & $\begin{array}{l}\text { follow- } \\
\text { up. }\end{array}$ & & & & & & \begin{tabular}{|l|} 
County \\
Council, \\
SCBCC \\
Sweden. 10.2 \\
Declared no \\
conflicts. \\
\end{tabular} & \\
\hline \multirow{2}{*}{$\begin{array}{l}\text { Ponce et } \\
\text { al., } 2012 \text {. }\end{array}$} & Yes & Yes & Unclear & Yes & No & Yes & Unclear & No & Yes & No & Neutral \\
\hline & $\begin{array}{l}\text { "Evaluated } \\
\text { the safety and } \\
\text { efficacy of an } \\
\text { intragastric } \\
\text { dual balloon } \\
\text { as an adjunct } \\
\text { to diet and } \\
\text { exercise in } \\
\text { obese patients } \\
\text { compared } \\
\text { with diet and } \\
\text { exercise } \\
\text { alone." }\end{array}$ & $\begin{array}{l}2.4 \\
\text { Sampling } \\
\text { unclear. }\end{array}$ & $\begin{array}{l}3.3 \\
\text { Concurrent } \\
\text { control } \\
3.4 \\
\text { Confounder } \\
\text { s not } \\
\text { accounted } \\
\text { for. }\end{array}$ & $\begin{array}{l}4.3 \\
\text { Unclear, } \\
\text { tables not } \\
\text { labelled } \\
\text { with } \\
\text { participan } \\
\text { t number. }\end{array}$ & $\begin{array}{l}\text { 5.1 No } \\
\text { blinding - } \\
\text { unable to } \\
\text { blind with } \\
\text { adverse } \\
\text { events } \\
\text { post } \\
\text { insertion } \\
\text { unmaskin } \\
\text { g } \\
\text { treatment } \\
\text { group - } \\
\text { p.292. }\end{array}$ & $\begin{array}{l}6.4 \text { Compliance } \\
\text { measured (food } \\
\text { journal). }\end{array}$ & $\begin{array}{l}7.4 \text { Not } \\
\text { reported. } \\
7.6 \text { Other } \\
\text { factors not } \\
\text { accounted } \\
\text { for. }\end{array}$ & $\begin{array}{l}8.1 \\
\text { Insufficien } \\
\text { t reporting. } \\
8.2 \\
\text { Inappropri } \\
\text { ate } \\
\text { statistical } \\
\text { methods. } \\
8.4 \text { Nil } \\
\text { intention } \\
\text { to treat. } \\
8.5 \text { No } \\
\text { adjustment } \\
\text { for } \\
\text { confounde } \\
\text { rs. }\end{array}$ & $\begin{array}{l}9.1 \\
\text { Discussion } \\
\text { included. } \\
9.2 \\
\text { Limitation } \\
\text { s } \\
\text { discussed. }\end{array}$ & $\begin{array}{l}10.1 \text { Funded } \\
\text { by Reshape } \\
\text { medical. } 10.2 \\
1 \text { author is a } \\
\text { consultant for } \\
\text { the funder. }\end{array}$ & \\
\hline \multirow{2}{*}{$\begin{array}{l}\text { Reimao } \\
\text { et al., } \\
2018\end{array}$} & Yes & Yes & N/A & Yes & N/A & Yes & Yes & Unclear & Yes & Unclear & Positive \\
\hline & $\begin{array}{l}\text { "To evaluate } \\
\text { the effects of } \\
\text { IGB in } \\
\text { overweight or } \\
\text { class } 1 \text { obese } \\
\text { patients, by } \\
\text { analysing } \\
\text { body } \\
\text { composition } \\
\text { and quality of } \\
\text { life". -p1 } 1806 \text {. }\end{array}$ & $\begin{array}{l}2.4 \\
\text { Consecutiv } \\
\text { e sample. }\end{array}$ & $\begin{array}{l}\text { One group } \\
\text { in study. }\end{array}$ & $\begin{array}{l}4.210 \% \\
\text { attrition - } \\
\text { withdraw } \\
\text { als } \\
\text { described } \\
\text {. }\end{array}$ & & $\begin{array}{l}6.4 \text { Compliance } \\
\text { not reported. }\end{array}$ & & $\begin{array}{l}8.3 \mathrm{QoL} \\
\text { data } \\
\text { reporting } \\
\text { method } \\
\text { unclear. } \\
8.4 \text { Nil } \\
\text { intention } \\
\text { to treat. } \\
8.5 \text { No } \\
\text { adjustment } \\
\text { s made. } \\
\end{array}$ & & $\begin{array}{l}10.1 \text { funding } \\
\text { not reported. } \\
10.2 \text { Declared } \\
\text { no conflict. }\end{array}$ & \\
\hline \multirow{2}{*}{$\begin{array}{l}\text { Raftopou } \\
\text { los et al., } \\
2017 .\end{array}$} & Unclear & Yes & N/A & Yes & N/A & Yes & Unclear & Unclear & Yes & No & Neutral \\
\hline & $\begin{array}{l}\text { "This study } \\
\text { aims to report } \\
\text { on } 12 \text {-month } \\
\text { safety and }\end{array}$ & $\begin{array}{l}2.4 \\
\text { "Unselecte } \\
\text { d sample" } \\
\text { Recruitmen }\end{array}$ & $\begin{array}{l}\text { One group } \\
\text { in study. }\end{array}$ & $\begin{array}{l}4.2 \mathrm{No} \\
\text { patient } \\
\text { drop-outs } \\
\text { or }\end{array}$ & & $\begin{array}{l}\text { 6.3 3-4 months } \\
\text { (time differed). } \\
\text { 6.4 Exposure } \\
\text { measured. }\end{array}$ & $\begin{array}{l}7.2 \\
\text { IWQOL- } \\
\text { Lite used. } \\
7.31-y . \\
\end{array}$ & $\begin{array}{l}8.2 \\
\text { IWQOL } \\
\text { score } \\
\text { decrease } \\
\end{array}$ & & $\begin{array}{l}10.1 \text { Funding } \\
\text { not reported. } \\
10.2 \text { One } \\
\text { author } \\
\end{array}$ & \\
\hline
\end{tabular}

This is a post-peer-review, pre-copyedit version of an article published in Obesity Surgery. 


\begin{tabular}{|c|c|c|c|c|c|c|c|c|c|c|c|}
\hline & $\begin{array}{l}\text { efficacy } \\
\text { outcomes." } \\
\text { Intervention } \\
\& \text { population } \\
\text { not reported } \\
(1.1,1.3) .\end{array}$ & $\begin{array}{l}\mathrm{t} \text { method } \\
\text { not } \\
\text { described. }\end{array}$ & & $\begin{array}{l}\text { missing } \\
\text { data, 1 } \\
\text { patient } \\
\text { excluded- } \\
91 \% \\
\text { follow-up } \\
\text { rate. }\end{array}$ & & $\begin{array}{l}6.5 \text { Co- } \\
\text { interventions } \\
\text { described. }\end{array}$ & $\begin{array}{l}\text { 7.4 Not all } \\
\text { measureme } \\
\text { nts } \\
\text { described. }\end{array}$ & $\begin{array}{l}\text { referred to } \\
\text { significant } \\
\text { improvem } \\
\text { ent. } 8.5 \\
\text { Pearson } \\
\text { correlation } \\
\text { used to } \\
\text { assess } \\
\text { linear } \\
\text { relationshi } \\
\text { p. } 8.7 \text { No } \\
\text { power } \\
\text { calculation } \\
\text { completed } \\
\end{array}$ & & $\begin{array}{l}\text { received } \\
\text { consulting } \\
\text { fees from } \\
\text { Allurion } \\
\text { techonologies } \\
\text {. }\end{array}$ & \\
\hline \multirow{2}{*}{$\begin{array}{l}\text { Tayyem, } \\
\text { Atkinson } \\
\& \\
\text { Martin, } \\
2014 .\end{array}$} & Unclear & Unclear & N/A & No & N/A & No & No & Unclear & Unclear & Unclear & Neutral \\
\hline & $\begin{array}{l}\text { "Develop and } \\
\text { validate a new } \\
\text { bariatric } \\
\text { specific } 81- \\
\text { item self- } \\
\text { report } \\
\text { HRQOL } \\
\text { instrument } \\
\text { called the } \\
\text { Bariatric and } \\
\text { Obesity- } \\
\text { Specific } \\
\text { Survey } \\
\text { (BOSS)." } 1.3 \\
\text { Population not } \\
\text { reported }\end{array}$ & $\begin{array}{l}2.4 \\
\text { Sampling } \\
\text { method } \\
\text { unclear. }\end{array}$ & $\begin{array}{l}\text { One } \\
\text { endoscopic } \\
\text { group in } \\
\text { study. }\end{array}$ & $\begin{array}{l}4.2 \\
\text { follow-up } \\
\text { rate } 49 \%, \\
\text { reasons } \\
\text { described } \\
\dot{4.3} \\
\text { Unclear. }\end{array}$ & & $\begin{array}{l}\text { 6.1 Protocol not } \\
\text { described. } \\
6.3 \text { Not stated. } \\
6.4 \text { Therapy } \\
\text { exposure not } \\
\text { measured. } \\
\text { 6.5 Other } \\
\text { treatments not } \\
\text { described. }\end{array}$ & $\begin{array}{l}7.1 \\
\text { Outcomes } \\
\text { not stated. } \\
7.42 \text { weeks } \\
\text { not } \\
\text { sufficient. }\end{array}$ & $\begin{array}{l}8.1 \\
\text { Reported } \\
\text { appropriat } \\
\text { ely. } 8.2 \\
\text { Appropriat } \\
\text { e tests. } 8.3 \\
\text { p }<0.05 . \\
8.4 \mathrm{Nil} \\
\text { intention } \\
\text { to treat. } \\
8.5 \mathrm{No} \\
\text { adjustment } \\
\text { for } \\
\text { confounde } \\
\text { rs. }\end{array}$ & $\begin{array}{l}9.2 \\
\text { Limitation } \\
\text { s not } \\
\text { discussed. }\end{array}$ & $\begin{array}{l}10.1 \text { funding } \\
\text { not reported. } \\
10.2 \text { No } \\
\text { conflicts } \\
\text { declared. }\end{array}$ & \\
\hline \multirow{2}{*}{$\begin{array}{l}\text { Deliopou } \\
\text { lo et al., } \\
2013\end{array}$} & Yes & Unclear & Yes & Unclear & Yes & Yes & Yes & Yes & Yes & Yes & Neutral \\
\hline & $\begin{array}{l}\text { "To examine } \\
\text { the 6-month } \\
\text { outcome of } \\
\text { depression } \\
\text { status - } \\
\text { assessed by a } \\
\text { well- } \\
\text { recognised } \\
\text { psychological }\end{array}$ & $\begin{array}{l}2.1 \\
\text { Exclusion } \\
\text { criteria not } \\
\text { stated. } 2.3 \\
\text { BD-II, } \\
\text { BMI, sex. } \\
2.4 \\
\text { "consecuti } \\
\text { vely }\end{array}$ & $\begin{array}{l}3.2 \text { Non } \\
\text { depressed } \\
\text { versus } \\
\text { depressed } \\
\text { (grouped } \\
\text { into } \\
\text { severity). } \\
\text { 3.4 Chi- } \\
\text { square }\end{array}$ & $\begin{array}{l}4.2 \\
\text { Withdraw } \\
\text { als not } \\
\text { reported. } \\
4.3 \\
\text { Tables do } \\
\text { not state } \\
\text { number } \\
\text { of }\end{array}$ & $\begin{array}{l}5.1 " \text { The } \\
\text { Beck } \\
\text { Depressio } \\
\mathrm{n} \\
\text { Inventory } \\
\text { score was } \\
\text { used at } \\
\text { time } 0 \text { to } \\
\text { blindly }\end{array}$ & $\begin{array}{l}6.1 \text { Insufficient } \\
\text { information. } \\
6.3 \text { 6month. } \\
6.4 \text { Patient drop } \\
\text { out not discussed. } \\
6.6 \text { Co- } \\
\text { interventions } \\
\text { described. }\end{array}$ & $\begin{array}{l}7.1 \\
\text { Outcomes } \\
\text { stated. } \\
7.3 \\
6 \text { month. } \\
7.4 \\
\text { Standard/ } \\
\text { valid } \\
\text { measures. }\end{array}$ & $\begin{array}{l}8.1 \\
\text { Reported } \\
\text { appropriat } \\
\text { ely. } \\
8.3 \\
\text { p }<0.05 . \\
8.4 \mathrm{Nil} \\
\text { intent to } \\
\text { treat. }\end{array}$ & $\begin{array}{l}9.1 \\
\text { Discussed } \\
\text { findings. } \\
9.2 \\
\text { Limitation } \\
\mathrm{s} \\
\text { discussed. }\end{array}$ & $\begin{array}{l}10.1 \text { Funding } \\
\text { not reported, } \\
\text { author } \\
\text { contacted and } \\
\text { stated } \\
\text { reported no } \\
\text { funding } \\
\text { sourced. } 10.2\end{array}$ & \\
\hline
\end{tabular}




\begin{tabular}{|c|c|c|c|c|c|c|c|c|c|c|c|}
\hline & $\begin{array}{l}\text { measure, } \\
\text { namely the } \\
\text { Beck } \\
\text { Depression } \\
\text { Inventory in } \\
\text { all patients } \\
\text { treated by } \\
\text { intragastric } \\
\text { balloon.... } \\
\text { between } \\
\text { depressed and } \\
\text { non-depressed } \\
\text { individuals" - } \\
\text { p.669. }\end{array}$ & $\begin{array}{l}\text { present } \\
\text { obese } \\
\text { female } \\
\text { patients" } \\
100 \% \\
\text { female not } \\
\text { representati } \\
\text { ve. }\end{array}$ & $\begin{array}{l}\text { analyses } \\
\text { used to } \\
\text { account for } \\
\text { differences } \\
\text { in } \\
\text { confounders } \\
\text {. }\end{array}$ & $\begin{array}{l}\text { participan } \\
\text { ts in } \\
\text { analysis. }\end{array}$ & $\begin{array}{l}\text { discrimin } \\
\text { ate the } \\
100 \text { obese } \\
\text { women } \\
\text { into those } \\
\text { with an } \\
\text { absence } \\
\text { of } \\
\text { depressio } \\
\mathrm{n} \text { [score } \\
\text { from } 0 \text { to } \\
9, \mathrm{n}=35 \\
\text { patients] } \\
\text { and those } \\
\text { having } \\
\text { depressiv } \\
\mathrm{e} \\
\text { symptoms } \\
\text { of varying } \\
\text { severity } \\
\text { [score } \\
\text { from } 10 \\
\text { to } 63, \\
\text { n=65]." - } \\
\text { p.670. }\end{array}$ & & $\begin{array}{l}\text { 7.6 Chi } \\
\text { Square } \\
\text { analysis } \\
\text { for } \\
\text { depressed } \\
\text { group only } \\
\text { no analysis } \\
\text { of } \\
\text { confounder } \\
\text { s for non- } \\
\text { depressed } \\
\text { group. } \\
7.7 \text { Non- } \\
\text { depressed } \\
\text { group no } \\
\text { measure } \\
\text { for QoL } \\
\text { change. }\end{array}$ & $\begin{array}{l}8.5 \\
\text { multivariat } \\
\text { e analysis } \\
\text { used. }\end{array}$ & & $\begin{array}{l}\text { Declared no } \\
\text { conflicts. }\end{array}$ & \\
\hline \multirow{2}{*}{$\begin{array}{l}\text { Tayyem, } \\
\text { Obondo } \\
\text { Ali, } \\
2011 .\end{array}$} & Yes & Yes & N/A & Yes & N/A & Yes & Yes & Unclear & Yes & Unclear & Positive \\
\hline & $\begin{array}{l}\text { "Describe } \\
\text { short-term } \\
\text { outcome and } \\
\text { quality of life } \\
\text { (QoL) of } \\
\text { endoscopicall } \\
\text { y placed } \\
\text { gastric } \\
\text { balloon } \\
\text { (EPGB) and } \\
\text { laparoscopic } \\
\text { adjustable } \\
\text { gastric band } \\
\text { (LAGB)." } 1.3\end{array}$ & $\begin{array}{l}2.1 \\
\text { Inclusion } \\
\& \\
\text { exclusion } \\
\text { reported. } \\
2.3 \text { Age, } \\
\text { weight \& } \\
\text { comorbiditi } \\
\text { es } \\
\text { described. } \\
2.4 \\
\text { Convenien } \\
\text { ce sample. }\end{array}$ & $\begin{array}{l}\text { One } \\
\text { endoscopic } \\
\text { group in } \\
\text { study. }\end{array}$ & $\begin{array}{l}4.1 \\
\text { Follow- } \\
\text { up } \\
\text { described } \\
\text { time } \\
\text { point } \\
\text { unclear. } \\
4.2 \text { No } \\
\text { withdraw } \\
\text { als or } \\
\text { dropouts } \\
\text { reported. }\end{array}$ & $\begin{array}{l}\text { Blinding } \\
\text { N/A }\end{array}$ & $\begin{array}{l}6.4 \text { Compliance } \\
\text { not stated. }\end{array}$ & $\begin{array}{l}\text { 7.4 Not all } \\
\text { measureme } \\
\mathrm{nt} \\
\text { instrument } \\
\mathrm{s} \\
\text { described. } \\
7.6 \\
\text { Complicati } \\
\text { ons } \\
\text { measured. } \\
\text { Confounde } \\
\text { r stated - } \\
\text { orlistat } \\
120 \mathrm{mg}\end{array}$ & $\begin{array}{l}8.2 \text { No } \\
\text { discussion } \\
\text { of non- } \\
\text { parametric } \\
\text { variables. } \\
8.4 \text { Nil } \\
\text { intent to } \\
\text { treat. } \\
8.5 \text { No } \\
\text { adjustment } \\
\text { for } \\
\text { confounde } \\
\text { rs - } \\
\text { univariate }\end{array}$ & & $\begin{array}{l}\text { 10.1 Funding } \\
\text { not reported. } \\
10.2 \text { No } \\
\text { conflicts } \\
\text { declared. }\end{array}$ & \\
\hline
\end{tabular}




\begin{tabular}{|c|c|c|c|c|c|c|c|c|c|c|c|}
\hline & $\begin{array}{l}\text { Population not } \\
\text { stated. }\end{array}$ & & & & & & $\begin{array}{l}\text { taken } \\
3 \mathrm{x} / \text { day } \\
\text { prescribed } \\
\text { in pre- } \\
\text { therapy to } \\
\text { aid weight } \\
\text { loss -p.3. }\end{array}$ & $\begin{array}{l}\text { analysis } \\
\text { (Orlistat } \\
\text { not } \\
\text { accounted } \\
\text { for). }\end{array}$ & & & \\
\hline \multirow{2}{*}{$\begin{array}{l}\text { Thompso } \\
\text { n et al, } \\
2017\end{array}$} & Yes & Yes & Unclear & Yes & $\mathrm{N} / \mathrm{A}$ & Yes & Yes & Yes & Yes & No & Positive \\
\hline & $\begin{array}{l}\text { "To evaluate } \\
\text { the efficacy } \\
\text { and safety of } \\
\text { AspireAssist } \\
\text { for weight } \\
\text { management } \\
\text { in persons } \\
\text { who have } \\
\text { obesity." - } \\
\text { p.448. }\end{array}$ & $\begin{array}{l}2.4 \\
\text { Sampling } \\
\text { unclear } \\
\text { conducted } \\
\text { at } 10 \text { sites. }\end{array}$ & $\begin{array}{l}\text { 3.3Historic } \\
\text { al controls. } \\
3.4 \text { Changes } \\
\text { made to } \\
\text { treat } \\
\text { cardiometab } \\
\text { olic } \\
\text { conditions } \\
\text { by the } \\
\text { participants } \\
\text { primary care } \\
\text { physicians } \\
\text { (p.454) but } \\
\text { not } \\
\text { accounted } \\
\text { for in } \\
\text { analysis. }\end{array}$ & $\begin{array}{l}4.2 \\
\text { Withdraw } \\
\text { als } \\
\text { described } \\
<74 \% \text {. }\end{array}$ & $\begin{array}{l}5.1 \\
\text { Participan } \\
\text { ts not } \\
\text { blinded } \\
\text { due to the } \\
\text { nature of } \\
\text { study. } 5.2 \\
\text { Unclear if } \\
\text { data } \\
\text { collectors } \\
\text { blinded. }\end{array}$ & & $\begin{array}{l}7.5 \\
\text { Measurem } \\
\text { ent of } \\
\text { effect not } \\
\text { described. } \\
7.6 \text { Other } \\
\text { factors not } \\
\text { measured. }\end{array}$ & $\begin{array}{l}8.1 \text { No } \\
\text { discussion } \\
\text { of non- } \\
\text { parametric } \\
\text { variables } \\
\& \text { what } \\
\text { data is } \\
\text { presented } \\
\text { in the } \\
\text { statistical } \\
\text { analysis. } \\
\text { Mean, SD } \\
\text { reported in } \\
\text { tables \& } \\
\text { labelled. } \\
8.4 \\
\text { Modified } \\
\text { intention } \\
\text { to treat in } \\
\text { statistical } \\
\text { analysis \& } \\
\text { tables. } 8.5 \\
\text { No } \\
\text { multivariat } \\
\text { e analysis. } \\
8.7 \text { power } \\
\text { calculation } \\
\text { used. }\end{array}$ & & $\begin{array}{l}10.1 \text { Funded } \\
\text { by Aspire } \\
\text { Bariatrics. } \\
10.22 \text { authors } \\
\text { are employees } \\
\text { of Aspire } \\
\text { Bariatrics. }\end{array}$ & \\
\hline \multirow{2}{*}{$\begin{array}{l}\text { Fiorillo } \\
\text { et al., } \\
2020\end{array}$} & Yes & No & N/A & No & N/A & Yes & Yes & Unclear & Yes & Unclear & Neutral \\
\hline & & & & & & & & & & & \\
\hline
\end{tabular}

This is a post-peer-review, pre-copyedit version of an article published in Obesity Surgery. 


\begin{tabular}{|c|c|c|c|c|c|c|c|c|}
\hline & $\begin{array}{l}\text { "To compare } \\
\text { QoL after } \\
\text { ESG and LSG } \\
\text { using a } \\
\text { propensity } \\
\text { score } \\
\text { analysis". } 1.3 \\
\text { Population not } \\
\text { specified. }\end{array}$ & $\begin{array}{l}2.1 \text { No } \\
\text { exclusion. } \\
2.2 \text { Age, } \\
\text { sex, } \\
\text { comorbiditi } \\
\text { es. } 2.3 \\
\text { Consecutiv } \\
\text { e sample } \\
\text { but then } \\
\text { sample } \\
\text { exclusion } \\
\text { through } \\
\text { propensity } \\
\text { score } \\
\text { matching } \\
\text { (PSM). } \\
\text { Not } \\
\text { representati } \\
\text { ve. }\end{array}$ & $\begin{array}{l}\text { Only ESG } \\
\text { data } \\
\text { reviewed. }\end{array}$ & $\begin{array}{l}4.2 \\
\text { Reason } \\
\text { for } \\
\text { withdraw } \\
\text { al not } \\
\text { reported. } \\
51.5 \% \text { of } \\
\text { patients } \\
\text { followed } \\
\text { up \& } \\
\text { only } 27 \% \\
\text { included } \\
\text { in study } \\
\text { after } \\
\text { PSM. } \\
4.3 \text { Yes. }\end{array}$ & $\begin{array}{l}6.5 \text { No description } \\
\text { of co- } \\
\text { interventions. }\end{array}$ & $\begin{array}{l}7.1 \\
\text { Outcomes } \\
\text { described. }\end{array}$ & $\begin{array}{l}8.1 \\
\text { Inadequate } \\
\text { ly } \\
\text { described. } \\
8.2 \\
\text { Unclear- } \\
\text { Logistic } \\
\text { regression } \\
\text { not } \\
\text { appropriat } \\
\text { e. } 8.3 \text { p- } \\
\text { value } \\
\text { reported. } \\
8.4 \text { Nil } \\
\text { intent to } \\
\text { treat. } 8.5 \\
\text { Logistic } \\
\text { regression. }\end{array}$ & $\begin{array}{l}10.1 \text { Funding } \\
\text { source not } \\
\text { reported. } 10.2 \\
\text { Authors } \\
\text { declare that } \\
\text { they have no } \\
\text { conflict of } \\
\text { interest. }\end{array}$ \\
\hline
\end{tabular}


Table S4: GRADE assessment of the confidence in the body of evidence

Question: What is the effect of endoscopic bariatric procedures on post-procedure QoL and mental health of adult patients? (in comparison to pre-procedural QoL and mental health).

\begin{tabular}{|c|c|c|c|c|c|c|c|c|c|c|}
\hline \multicolumn{7}{|c|}{ Certainty assessment } & \multicolumn{2}{|c|}{ № of patients } & \multirow{2}{*}{\begin{tabular}{|l|} 
Effect \\
Absolute \\
$(95 \%$ CI $)$
\end{tabular}} & \multirow[b]{2}{*}{ Certainty } \\
\hline $\begin{array}{l}\text { № of } \\
\text { studies }\end{array}$ & Study design & $\begin{array}{l}\text { Risk of } \\
\text { bias }\end{array}$ & Inconsistency & Indirectness & Imprecision & $\begin{array}{l}\text { Other } \\
\text { considerations }\end{array}$ & $\begin{array}{l}\text { Pre- } \\
\text { procedure }\end{array}$ & $\begin{array}{l}\text { Post- } \\
\text { procedure }\end{array}$ & & \\
\hline \multicolumn{11}{|c|}{$\begin{array}{l}\text { Quality of Life (QOL) (follow up: range } 4 \text { months to } 76 \text { months; assessed with: SF-36, EQ-5D, GIQLI, IWQOL-BREF, IWQOL-Lite, SF-12; Scale } \\
\text { from: } 0 \text { to 100) }\end{array}$} \\
\hline 19 & $\begin{array}{l}\text { Observational } \\
\text { studies }\end{array}$ & Seriousa & not serious & not serious & not serious & $\begin{array}{l}\text { Strong } \\
\text { association }\end{array}$ & 768 & 654 & $\begin{array}{l}\text { SMD } 0.83 \\
\text { SD higher } \\
(0.67 \text { higher } \\
\text { to } 0.99 \\
\text { higher })\end{array}$ & $\begin{array}{l}\oplus \oplus \bigcirc \bigcirc \\
\mathrm{LOW}\end{array}$ \\
\hline \multicolumn{11}{|c|}{ Mental Health (follow up: mean 6 months; assessed with: BDI, SF-36 Anxiety, HDAS-A, HDAS-D; Scale from: 7.9 to 84) } \\
\hline 8 & $\begin{array}{l}\text { Observational } \\
\text { studies }\end{array}$ & $1 \begin{array}{l}\text { Very } \\
\text { seriousb }\end{array}$ & Very seriousc & not serious & Seriousa,c & $\begin{array}{l}\text { Strong } \\
\text { association }\end{array}$ & 409 & 363 & $\begin{array}{l}\text { SMD } 0.41 \\
\text { SD higher } \\
(0.23 \text { higher } \\
\text { to } 0.6 \\
\text { higher })\end{array}$ & $\begin{array}{l}\oplus \bigcirc \bigcirc \bigcirc \\
\text { Very LOW }\end{array}$ \\
\hline
\end{tabular}

CI: Confidence interval; SMD: Standardised mean difference; SD: Standard deviation

Explanations

a. Primary research outcome and patient centred outcome.

b. Confounding variables not accounted for. 
c. Heterogeneity was $92 \%$ indicating serious imprecision and may have been the result of the type of tool used to assess mental health and/or the amount of multidisciplinary support provided to patients.

1. World Health Organization. Obesity and overweight. . Available from: https://www.who.int/news-room/fact-sheets/detail/obesity-andoverweight. Published 2018. Acessed November 11, 2019.

2. Guedes, E., et al., Impact of 6 months of treatment with intragastric balloon on body fat and quality of life in obese individuals with metabolic syndrome. Health and Quality of Life Outcomes, 2017. 15(1): p. 1-6.

3. Sierżantowicz, R., et al., Effect of BMI on quality of life and depression levels after bariatric surgery. Advances in clinical and experimental medicine : official organ Wroclaw Medical University, 2017. 26(3): p. 491.

4. Angrisani, L., et al., IFSO Worldwide Survey 2016: Primary, Endoluminal, and Revisional Procedures. Obesity Surgery, 2018. 28(12): p. 3783-3794.

5. Buchwald, H., et al., Bariatric surgery: A systematic review and meta-analysis. Jama-Journal Of The American Medical Association, 2004. 292(14): p. 1724-1737. 
6. Regan, J., et al., Early Experience with Two-Stage Laparoscopic Roux-en-Y Gastric Bypass as an Alternative in the Super-Super Obese Patient. Obesity Surgery, 2003. 13(6): p. 861-864.

7. Schwartz, A., L. Etchechoury, and D. Collet, Outcome after laparoscopic gastric bypass for super-super obese patients. Journal of Visceral Surgery, 2013. 150(2): p. 145-149.

8. Villamere, J., et al., Body mass index is predictive of higher in-hospital mortality in patients undergoing laparoscopic gastric bypass but not laparoscopic sleeve gastrectomy or gastric banding. American Surgeon, 2014. 80(10): p. 1039-1043.

9. Behary, J. and V. Kumbhari, Advances in the Endoscopic Management of Obesity. Gastroenterology Research and Practice, 2015.

\section{(2015): 1-9.}

10. Abu Dayyeh, B.K., et al., ASGE Bariatric Endoscopy Task Force systematic review and meta-analysis assessing the ASGE PIVI thresholds for adopting endoscopic bariatric therapies. Gastrointest Endoscopy, 2015. 82(3): p. 425-38.e5.

11. FDA (Food and Drug Adminitsration). Weight-Loss and Weight Management Devices. 2019 [cited 2020 11-03]; Available from: https://www.fda.gov/medical-devices/products-and-medical-procedures/weight-loss-and-weight-management-devices\#loss.

12. Familiari, P., et al., Transoral gastroplasty for morbid obesity: a multicenter trial with a 1-year outcome. Gastrointestinal Endoscopy, 2011. 74(6): p. 1248-1258.

13. Fiorillo, C., et al., 6-Month Gastrointestinal Quality of Life (QoL) Results after Endoscopic Sleeve Gastroplasty and Laparoscopic Sleeve Gastrectomy: A Propensity Score Analysis. Obes Surg, 2020. 
14. Jason, B. and K. Vivek, Advances in the Endoscopic Management of Obesity. Gastroenterology Research and Practice, 2015.

\section{5(2015).}

15. Saber, A.A., et al., Efficacy of First-Time Intragastric Balloon in Weight Loss: a Systematic Review and Meta-analysis of Randomized Controlled Trials. Obes Surg, 2017. 27(2): p. 277-287.

16. Spirou, D., J. Raman, and E. Smith, Psychological outcomes following surgical and endoscopic bariatric procedures: A systematic review. Obes Rev, 2020.

17. Yorke, E., et al., Intragastric Balloon for Management of Severe Obesity: a Systematic Review. Obes Surg, 2016. 26(9): p. $2248-2254$.

18. Fernandes, M.A.P., et al., Intragastric balloon for obesity. Cochrane Database of Systematic Reviews, 2007(1).

19. Szmulewicz, A., et al., Mental health quality of life after bariatric surgery: A systematic review and meta-analysis of randomized clinical trials. Clinical obesity, 2019. 9(1): p. e12290.

20. Mui, W., et al., Impact on Obesity-Related Illnesses and Quality of Life Following Intragastric Balloon. Obesity Surgery, 2010. 20(8): p. $1128-1132$.

21. NCE (National Institute for Health and Care Excellence) Obesity: identification, assessment and management. 2014.

22. Accardi, R., et al., Italian version of the laval questionnaire: Validity and reliability. Bariatric Surgical Practice and Patient Care, 2017. 12(3): p. 136-141. 
23. Moher, D., et al., Preferred reporting items for systematic reviews and meta-analyses: the PRISMA statement. Annals of internal medicine, 2009. 151(4): p. 264.

24. Google. Google Translate. Available from: https://translate.google.com/. Acessed November 11, 2019.

25. Bond University. Systematic Review Accelerator.; Available from: http://sr-accelerator.com/\#/. Acessed November 11, 2019.

26. EndNote [computer program]. Version X8, Web of Science Group. 2019.

27. Covidence systematic review software, Veritas Health Innovation, Melbourne, Australia. Available at www.covidence.org.

28. $\quad$ PLOTCON, WebplotDigitizer. 2017: Oakland, CA.

29. Academy of Nutrition \& Dietetics. Evidence Analysis Manual. Appendix 8: Quality Criteria Checklist: Primary Research Available from: https://www.andeal.org/evidence-analysis-manual Published 2016. Accessed October 28, 2019

30. Erika Paniago, G., et al., Impact of 6 months of treatment with intragastric balloon on body fat and quality of life in obese individuals with metabolic syndrome. Health and Quality of Life Outcomes, 2017. 15(1): p. 1-6.

31. The Cochrane Collaboration, Review Manager [computer program]. Version 5.3. 2014: Copenhagen: The Nordic Cochrane Centre; .

32. Higgins, J.P.T., Cochrane Handbook for Systematic Reviews of Interventions. 2nd ed. ed. Wiley Cochrane Ser., ed. J. Thomas. 2019, Newark: John Wiley \& Sons, Incorporated. 
33. Tayyem, R.M., C. Obondo, and A. Ali, Short-term outcome and quality of life of endoscopically placed gastric balloon and laparoscopic adjustable gastric band. Saudi journal of gastroenterology : official journal of the Saudi Gastroenterology Association, 2011. 17(6): p. 400.

34. Tayyem, R.M., J.M. Atkinson, and C.R. Martin, Development and validation of a new bariatric-specific health-related quality of life instrument "bariatric and obesity-specific survey (BOSS)". Journal of postgraduate medicine, 2014. 60(4): p. 357.

35. Guedes, E., et al., Impact of a 6-month treatment with intragastric balloon on body composition and psychopathological profile in obese individuals with metabolic syndrome. Diabetology and Metabolic Syndrome, 2016. 8(1): p. 1-7.

36. Raftopoulos, I. and A. Giannakou, The Elipse Balloon, a swallowable gastric balloon for weight loss not requiring sedation, anesthesia or endoscopy: a pilot study with 12-month outcomes. Surgery for Obesity and Related Diseases, 2017. 13(7): p. 1174-1182.

37. Reimão, S., et al., Improvement of Body Composition and Quality of Life Following Intragastric Balloon. Obesity Surgery, 2018. 28(6): p. $1806-1808$.

38. Ponce, J., B.B. Quebbemann, and E.J. Patterson, Prospective, randomized, multicenter study evaluating safety and efficacy of intragastric dual-balloon in obesity. Surgery for Obesity and Related Diseases, 2013. 9(2): p. 290-295.

39. Machytka, E., et al., Elipse, the first procedureless gastric balloon for weight loss: a prospective, observational, open-label, multicenter study. Endoscopy, 2017. 49(2): p. 154. 
40. Guedes, M.R., et al., Changes in Body Adiposity, Dietary Intake, Physical Activity and Quality of Life of Obese Individuals Submitted to Intragastric Balloon Therapy for 6 Months. Obesity surgery, 2019. 29(3): p. 843.

41. Alfredo, G., et al., Long-term multiple intragastric balloon treatment — a new strategy to treat morbid obese patients refusing surgery: Prospective 6-year follow-up study. Surgery for Obesity and Related Diseases, 2014. 10(2): p. 307-311.

42. Fuller, N., et al., An intragastric balloon in the treatment of obese individuals with metabolic syndrome: A randomized controlled study. Obesity, 2013. 21(8): p. 1561-1570.

43. Deliopoulou, K., et al., The Impact of Weight Loss on Depression Status in Obese Individuals Subjected to Intragastric Balloon Treatment. Obesity Surgery, 2013. 23(5): p. 669-675.

44. Castro, M., et al., Efficacy, Safety, and Tolerance of Two Types of Intragastric Balloons Placed in Obese Subjects: A Double-Blind Comparative Study. Obesity Surgery, 2010. 20(12): p. 1642-1646.

45. Ahmed, H.O. and R.F. Ezzat, Quality of life of obese patients after treatment with the insertion of intra-gastric balloon versus Atkins diet in Sulaimani Governorate, Kurdistan Region, Iraq. Annals of medicine and surgery (2012), 2018. 37: p. $42-46$.

46. Christopher, C.T., et al., Percutaneous Gastrostomy Device for the Treatment of Class II and Class III Obesity: Results of a Randomized Controlled Trial. The American Journal of Gastroenterology, 2016. 112(3).

47. Norén, E. and H. Forssell, Aspiration therapy for obesity; a safe and effective treatment. BMC obesity, 2016. 3(1). 
48. Moreno, C., et al., Transoral gastroplasty is safe, feasible, and induces significant weight loss in morbidly obese patients: results of the second human pilot study. Endoscopy, 2008. 40(5): p. 406.

49. Marinos, G., et al., Weight loss and improved quality of life with a nonsurgical endoscopic treatment for obesity: clinical results from a 3- and 6-month study. Surgery for Obesity and Related Diseases, 2014. 10(5): p. 929-934.

50. Lindekilde, N., et al., The impact of bariatric surgery on quality of life: a systematic review and meta-analysis. 2015. p. 639-651.

51. Dawes, A.J., et al., Mental Health Conditions Among Patients Seeking and Undergoing Bariatric Surgery: A Meta-analysis. JAMA, 2016. 315(2): p. 150.

52. Canetti, L., E. Bachar, and O. Bonne, Deterioration of mental health in bariatric surgery after 10 years despite successful weight loss. European journal of clinical nutrition, 2016. 70(1): p. 17.

53. Marshall, S., et al., Does intensive multidisciplinary intervention for adults who elect bariatric surgery improve postoperative weight loss, comorbidities, and quality of life? A systematic review and meta-analysis Obesity Reviews, 2020. In Press.

54. Mechanick, J.I., et al., Clinical practice guidelines for the perioperative nutrition, metabolic, and nonsurgical support of patients undergoing bariatric procedures-2019 update: cosponsored by American Association of Clinical Endocrinologists/American College of Endocrinology, The Obesity Society, American Society for Metabolic \& Bariatric Surgery, Obesity Medicine Association, and American Society of Anesthesiologists. Surgery for Obesity and Related Diseases, 2019.

55. Cuijpers, P., et al., Pre-post effect sizes should be avoided in meta-analyses. Epidemiology and psychiatric sciences, $2017.26(4)$ : p. 364. 
56. Von Elm, E., et al., The Strengthening the Reporting of Observational Studies in Epidemiology (STROBE) statement: guidelines for reporting observational studies. Preventive Medicine, 2007. 45(4): p. 247-251.

57. Allergan. Intragastric Balloon System—Directions For Use (DFU). 2011; Available from: http://www.allergan.com.au/Products/Overview.aspx.

58. Beck, A.T., et al., An inventory for measuring depression. Archives of general psychiatry, 1961. 4: p. 561.

59. Beck, A.T., R.A. Steer, and G.K. Brown, Beck Depression Inventory - Second Edition (BDI-II). 1996.

60. EuroQol Research Foundation. EQ-5D-5L User Guide. 2019 [cited 2020 10.02]; Available from: https://euroqol.org/publications/userguides.

61. Eypasch, E., et al., Gastrointestinal Quality of Life Index: development, validation and application of a new instrument. Br J Surg, 1995. 82(2): p. 216-22.

62. Zigmond, A.S. and R.P. Snaith, The Hospital Anxiety and Depression Scale. Acta Psychiatrica Scandinavica, 1983. 67(6): p. 361-370.

63. Organization, W.H. WHO Quality of Life-BREF (WHOQOL-BREF). n.d; Available from:

https://www.who.int/substance_abuse/research_tools/whoqolbref/en/.

64. Kolotkin, R. IWQOL-Lite Asessing the impact of weight on quality of life in adults. 2017; Available from: https://www.qualityoflifeconsulting.com/iwqol-lite.html. 
65. Kolotkin, R.L., S. Head, and A. Brookhart, Construct validity of the impact of weight on quality of life questionnaire. Obesity research, 1997(5): p. 434-441.

66. Ware, J.E., M. Kosinski, and S.D. Keller, A 12-Item Short-Form Health Survey: Construction of Scales and Preliminary Tests of Reliability and Validity. Medical Care, 1996. 34(3): p. 220-233.

67. J., W., et al. SF-36 Health Survey Manual and Interpretation Guide. 1993. 
